\title{
Method to obtain homozygous introgressing segments in Drosophila to identify the presence of hybrid sterility genes of the reproductive isolation
}

Francisco García-Franco ( $\nabla$ ffranco68@hotmail.com )

Universidad Autónoma Metropolitana https://orcid.org/0000-0002-3424-5271

Lilian Barandica-Cañon

Universidad de Cartagena

Ezel Galindo-Pérez

Universidad Autónoma Metropolitana

Martha Martínez García

Universidad Nacional Autónoma de México

Blanca Chávez-Sandoval

Universidad Autónoma Metropolitana

\section{Biological Sciences - Article}

Keywords: Synmorphic Species, Polygenic Chromosome Asynapses, Bioinformatic Analysis, Crossing Strategies

Posted Date: November 19th, 2020

DOl: https://doi.org/10.21203/rs.3.rs-110613/v1

License: (c) (1) This work is licensed under a Creative Commons Attribution 4.0 International License. Read Full License 
1 Method to obtain homozygous introgressing segments in Drosophila to identify the presence of hybrid sterility genes of the reproductive isolation

\section{Corresponding authors: *ffranco68@hotmail.com and *blanchavez29@gmail.com}

\section{ABSTRACT}

Here, we present for the first time, a method to generate homozygous segmental introgressions, by means of crosses between a pair of synmorphic species. The introgressions were monitored by the cytogenetic method of polygenic chromosome asynapses. Later the introgressions were evaluated in their capacity to produce sterility in segmental males. Also, the smallest segment with the capacity to produce sterility in segmental males was mapped by in situ hybridization of polythene chromosomes, using 8 sequences of BACs clones as probes. Finally, a bioinformatic analysis was carried out to identify the presence of particular genes. From 2 parental strains, $D$. buzzatii and $D$. koepferae, 6 simple segmental hybrid lines were generated, whose introgressing segments are distributed along chromosome 4 of these species. From the 6 simple segmental lines and by means of a new crossing strategy, the 6 respective homozygous segmental hybrid offspring were obtained, each of them carrying a specific homozygous introgression. None of the 6 heterozygous introgressions was capable of producing sterility in segmental males, while 4 of the same homozygous introgressions produced total sterility in segmental males, including in this group the two smallest introgressive segments, one of $5.03 \%$ and the other $7.87 \%$ with respect to the total length of chromosome 4, which are located in the region F2 to F4 of the standard cytological map based on polythene chromosomes of the Drosophila Repleta group. In situ hybridization, using 8 clones from contig 1065 located along the F2 to F4 region of the physical map of D. buzzattii constructed in BACs, confirmed the precise location of the 6 clones in the chromosomal region F2 to F4 of chromosome 4 of the polygenic chromosomes of both D. buzzatii and D. mojavensis. The bioinformatic analysis of the F2 to F4 region, using the complete genetic sequence of the contig 1065 of $\mathrm{D}$. buzzatti shows the presence of two predicted genes in the genomic map of D. buzzatii (g.1313.t1 and g.1314.t1), and the orthologous association of these 2 genes both with the D. moj_GI22766 gene of D. mojavensis and with the Trivet gene of D. melanogaster.

\section{INTRODUCTION}

One long-standing issue of debate in speciation theory concerns the number of genes that are necessary for speciation to occur.

In Drosophila, it is suggested that the density of sterility factors may be of the order of one per 1 percent of the genome and, by extrapolation, a minimum of 40 of these loci has been estimated on the $X$ chromosome, which gives an estimate of the sterility genes between D. simulans and D. mauritiana of the order of 100 (Wu, 1994) (Palopoli, 1994). Under the classic Bateson-Dobzhansky-Muller (BDM) model, speciation requires changes in at least two genes (Nei and Zhang, 1998) (Orr, 2005) (Rieseberg and Blackman, 2010) (Nosil and Schluter, 2011) (Blackman, genes 2016) (Cooper, 2016) but there are several examples of empirical evidence for the determination of mating traits by a single locus, Allen Orr (1991), in his model demonstrates the possibility of single-gene speciation is possible if the character causing reproductive isolation is maternally inherited. While Gregorius (1992), does it with a model where he involves three alleles and the speciation by the reinforcement. For their part, Yamamichi and Sasaki (2013), propose that the pleiotropy of a single gene on delayed inheritance and dominance is capable of allowing speciation. In the same way, as Phadnis and Orr (2009), describe the effect of a single gene, both on hybrid sterility and on the distortion of segregation.

Genes responsible for intraspecific gene sterility (Rieseberg and Blackman, 2010) show an easily recognizable, clear-cut segregation, and are generally recessive. Experiments on genomic fragment introgression from one species into another suggest two possible 
types of action: 1) additive with a threshold effect (Naveira, et al., 1984) (Naveira \& Fontdevila, 1998) and 2) epistatic effects between conspecific genes in a heterospecific background (Palopoli, 1994); (Presgraves, 2007). Both architectures are polygenic, they do not exclude the presence of genes with greater effects with strong or additive effects, and that when identified can be cloned (Ting, et al., 1998), supporting the view that alleles of large effect that distinguish species may sometimes reflect the accumulation of multiple mutations of small effect at select genes (McGregor, et al., 2007). And Feller et al, 2020, found that several of the characters selected for reproductive isolation are assigned to a region of significant QTLs, supporting the theory of the presence of major effect loci.

Many researchers seek to do identify individual genes from reproductive isolation scattered throughout the Drosophila genome, and they do so by examining F2 backcross hybrids and high resolution analysis between closely related species (Perez and Wu, 1995) (Coyne and Orr, 1998) (Palopoli and Wu, 1994). In addition to genomic studies compared between the sequenced species of the genus melanogaster (Song et al., 2011) (Drosopgila 12 genome consortium, 207).

The Drosophila repleta group is an array of about 100 cactophilic species endemic to the New World, many of them cactophilic species living in the deserts and arid zones of the American continent and has become a model group for studies of ecological adaptation and speciation for over 70 years (Ruiz et al., 1982) (Fontdevila, et al., 1982) (Naveira, et al., 1984) (Fontdevila, et al., 1988) (Santos, et al., 1989) (Naveira \& Fontdevila, 1991) (Ruiz and Wasserman 1993) (Oliveira et al., 2012).

The buzzatii cluster consists of the seven cactophilic species, $D$. antonietae, $D$. borborema, $D$. buzzatii, $D$. gouveai $D$. koepferae, $D$. serido y $D$. seriema.

They are closely related species belonging to the buzzatii complex (repleta group). This species with various degrees of evolutionary divergence (Rodríguez-Trelles, et al., 2000); (Durando, et al., 2000) (Manfrin et al., 2001) which generates a broad spectrum of reproductive interactions among them.

They are closely related species belonging to the buzzatii complex (repleta group). This species has various degrees of evolutionary divergence (Rodríguez-Trelles, et al., 2000); (Durando, et al., 2000) which generates a broad spectrum of reproductive interactions among them. Recently Hurtado et al., 2019, analyzed the phylogeny of this group using a large transcriptomic dataset, $D$. buzzatii split from the ancestor of the $D$. serido sibling set approximately $1.5 \mathrm{Myr}$ ago, the same divergence time between $D$. buzzatti and $D$. koepferae, forming twoo different clades. The radiation of the remaining six species seems to be extremely recent, < $0.5-1$ Myr ago.

Considering the replacement rates of 6.8531029 per site per year for mtDNA, the estimated age for the separation of the clades comprised by $D$. koepferae and $D$. buzzatti from the Pve remaining $D$. buzzatti cluster species is approximately 6-12 Myr, whereas separation of the clades composed of $D$. sp.D and $D$. seriema, D. borborema, $D$. serido, and $D$. sp. B, is estimated at

3-6 Myr (Manfrin et al., 2001).

The divergence time estimates revealed that the buzzatii cluster is very young, with the first split that separates $D$. buzzatii occurring about $1.5 \mathrm{Myr}$ ago and the remaining species diverging within the last $\sim 0.5 \mathrm{Myr}$ (Hurtado et al, 2019).

Among those species, $D$. koepferae females can be crossed with $D$. buzzatii males under laboratory conditions (Naveira \& Fontdevila, 1986) (Machado, 2006) producing sterile hybrid males and fertile hybrid females, although reciprocal crosses never produce offspring. 


\subsection{EVALUATION OF CHROMOSOMAL SEGMENTS IN HOMOZYGOSITY}

\subsubsection{Standard cytological map of chromosome 4 for Drosophila replete group}

A) The cytological physic map for both $D$. koepferae and $D$. buzzati have the same polytene karyotype as species of the Drosophila repleta group consisting of six chromosomes.

Each chromosome is divided into cytological intervals, identified by capital letters ( $\boldsymbol{A}$ to $\boldsymbol{H}$ ). Each interval contains a specific amount of subintervals identified by numbers ( 1 to 5). Each subinterval is divided by a series of bands, identified by lowercase letters ( $a$ to h), in alphabetical order from telomere to centromere (Wharton, 1942), (Ruiz et al., 1982) (Naveira et al., 1986) (Schaeffer et al., 2008) see figure 1. The symbols of letters and numbers describe the localization and length of each chromosomal segment according to the cytological map figure 1.

Figure 1. A) Cytological map of polytene chromosome 4 of Drosophila Repleta group species. The vertical lines divide each cytogenetic interval $(\boldsymbol{A}$ to $\boldsymbol{H})$ into subintervals identified by Arabic numerals 1 to 5 . The arrows show one example of the classification of bands identified by lowercase letters ( $\boldsymbol{a}$ to $\boldsymbol{h}$ ) to subinterval 4 into the cytological interval $\boldsymbol{B}$.

\subsubsection{Method for genetic markers by chromosomal asynapsis}

Each Drosophila culture produced from cross and backcrosses, was analyzed from a sample of six to ten third instar larvae. Each offspring were genotyped based on the presence of genetic markers known as chromosomal asynapsis, each chromosomal segments introgressed produce one asynapsis is formed by an incomplete pairing of chromatin fibers in the area of union between a pair of homologous chromosomes, exclusively at sites where introgression has been successful. The procedure to identify chromosomal asynapsis of polytene chromosomes is performed by the standard squashing method: the salivary glands from a third instar Drosophila larva are extracted in 45 percent acetic acid and placed on a slide with a drop of standard dye solution lactoaceto-orcein. This preparation is sandwiched between a cover glass, placed between a paper-towel and crushed with the thumb tip to release the gland.

The length and location of the introgressing segment in the cytological map indicate each specific asynapsis. Each asynapsis segregates according to Mendel's laws, representing the genotypes of male segmental hybrids and their offspring, further allowing inference on the parent's genotype. The absence of asynapsis in the chromosomes of all the cells present in a squash preparation indicates that there was no integration of any chromosomal segment. When this happens in the squash preparations of at least 6 different larvae of a culture, then we consider that this offspring is of the genotype of the parental type without introgressions polytene chromosomes and allow them to extend over the surface of the cover glass. Finally, the preparation is observed under the microscope and its image captured with an adapted digital camera (Naveira et al., 1986) (Henderson, 2004). 


\subsubsection{Initial strains}

The $D$. buzzatii Bu-28 strain -designated $D b$ with genotype bu/bu- originated in a sample collected from a natural population at Los Negros, Bolivia in 1982; the strain $D$. koepferae KO-2, designated $D k$ with genotype ko/ko, was collected in December 1979 at the Sierra de San Luis, Argentina. The F1 progeny from each strain was placed in a population cage, later maintained in mass cultures at $25^{\circ} \mathrm{C}$ at the Universidad Autónoma de Barcelona Drosophila fly strain collection.

Table 1. Initial strain

\begin{tabular}{|c|c|c|c|c|}
\hline Initial strain & Specie & Genotype & Origin & Obtained \\
\hline Bu-28 & $\begin{array}{c}\text { Drosophila } \\
\text { buzzatii }\end{array}$ & bu/bu & $\begin{array}{c}\text { Los Negros, } \\
\text { Bolivia (1982) }\end{array}$ & Universidad Autónoma de \\
KO-2 & $\begin{array}{l}\text { Drosophila } \\
\text { koepferae }\end{array}$ & ko/ko & $\begin{array}{l}\text { Sierra de San } \\
\text { Luis, Argentina. } \\
\text { (1979). }\end{array}$ & strain collection. \\
\hline
\end{tabular}

Each strains were derived from a single wild inseminated female (isoline), taken from a respective population cage founded by the combination of $\mathrm{F} 1$ progenies of approximately 60 wild-inseminated females (isolines) collected. Both strains were kept by mass culturing thereafter.

\subsubsection{General conditions for crosses}

All $D b$ specimens were 7 days old, whereas segmental specimens were between 5 to 8 days old. For all crosses, parental flies were transferred every 5 days to a new flask with fresh feeding medium and maintained at $25^{\circ} \mathrm{C}$ on a 12:12 light: dark regime. During specimen manipulation, temperature never exceeded $25^{\circ} \mathrm{C}$. We performed cytogenetic analysis of at least 9 larvae from the offspring of each cross to look for introgressions and infer the parental genotype.

For all type crosses (individual, massal or backcrosses) to obtain the Drosophila segmental lines as well as to test and reciprocal crosses, we used randomly selected males and females.

\subsubsection{Method to obtaining single segmental lines in heterozygous state}

To obtain segmental hybrid lines of Drosophila buzzatii and Drosophila koepferae, it was necessary to follow a strict crossing scheme (Naveira et al., 1986, García-Franco 2020) that is summarized below in Figure 2. It is also necessary to consider, among those species, $D$. koepferae females can be crossed with $D$. buzzatii males under laboratory conditions producing sterile hybrid males and fertile hybrid females, although reciprocal crosses never produce offspring. And also, the genetic recombination only happens during meiosis in Drosophila females, allowing the appearance of new chromosomal arrangements (Naveira \& Fontdevila, 1986) (Machado, 2006).

On the other hand, it is also important to bear in mind that each new offspring is analyzed for the presence of cromosomal asynapsis to identify the type of introgressions present and thus be able to characterize and select them according to their segmental genotype. 1) Parental cross: 5 mass type crosses of 50 male flies of the Bu-28 parental strain of $D$. buzzatii with 50 female flies of the other parental strain KO-2 of $D$. koepferae, which will produce 5 fully hybrid offspring type $\mathrm{F} 1$.

2) Backcross $1\left(B_{1}\right)$ : five mass type crosses of each of five $F 2$ offspring, between 50 females of the hybrid offspring F1 with 50 parental males $\mathrm{Db}$, which will give rise to the twenty five recombinant cultures of multi-segmental offspring designates as F2.

3) Backcross $2\left(\mathrm{BC}_{2}\right)$ : five individual-type crosses of each of twenty five offspring $\mathrm{F} 2$, using 1 female of the F2 segmental offspring with 1 male $\mathrm{Db}$. Thanks to genetic recombination, these 125 new cultures produce more chromosomal arrangements and therefore new types of segments, therefore it is called multi-segmental F3 offspring. 
4) Backcross $3\left(\mathrm{BC}_{3}\right)$ : represents the first selection of 20 introgression-bearing offspring on chromosomal 4. Perform five individual-type crosses of each of twenty F3 cultures selected for the presence of introgressions on chromosome 4. Using 1 female of the selected F3 segmental offspring with 1 male $D b$. This offspring is designated as F4. 5 -14) Backcrosses 4 to $12\left(\mathrm{BC}_{4}-\mathrm{BC}_{13}\right)$ : They represent a series of 10 backcrosses in sequence to continue producing new chromosomal arrangements in each new offspring, until obtaining the introgressions of interest and being able to select them. Each one similar to BC3, where 20 cultures of each new offspring are selected for having introgressions on chromosome 4.

This new offspring (F5 to F14) are characterized by being multi-segmental and introgressive on chromosome 4.

15-24) Backcross 14 to $20\left(\mathrm{BC}_{14}-\mathrm{BC}_{20}\right)$ : They represent a series of 10 backcrosses in sequence to continue producing new chromosomal arrangements in each new offspring, until obtaining specific introgressions of interest and being able to select them. Each one similar to $\mathrm{BC}_{14}$, where 20 cultures of each new offspring are selected for having specific and single introgressions of interest on chromosome 4.

These new offspring (F15 to F24) are characterized by carrying specific single introgressions of interest exclusively on chromosome 4.

25) Backcross $24\left(\mathrm{BC}_{24}\right)$ : To keep the introgressions stable, the following backcrosses are now performed using the segmental male, since in Drosophila the males do not recombine during meiosis.

Selection of offspring with introgressions of interest on chromosome 4. Perform 3 individual-type crosses of each of twenty F25 cultures selected for the presence of specific introgressions on chromosome 4. Using one male of the selected F14 segmental offspring with 1 female $\mathrm{Db}$. This new offspring F25 are characterized by being specific single segmental on chromosome 4. From now on, the backcrosses will be carried out in this direction, the male of each new segmental offspring specific $\mathrm{x}$ female $D b$. Each of these selected offspring for their specific chromosomal segment of introgression represent stock lines in maintenance. 


\begin{tabular}{|c|c|c|c|c|}
\hline No. & $\begin{array}{l}\text { Parents' } \\
\text { generation }\end{array}$ & $\begin{array}{l}\text { Type of } \\
\text { croos }\end{array}$ & Cross description & Offspring \\
\hline 1 & Parental & Mass & $\left(50 \%\right.$ Dko $\left.\times 500^{\prime \prime} D b\right) \times 5$ & F1 \\
\hline 2 & $\mathrm{BC}_{1}$ & Mass & $\left(50 \% \mathrm{~F} 1 \times 500^{x} D b\right) \times 5[5]$ & F2 \\
\hline 3 & $\mathrm{BC}_{2}$ & Individual & $\left(19 \mathrm{~F} 2 \times 10^{\pi} \mathrm{Db}\right) \times 25[5]$ & F3 \\
\hline 4 & $\mathrm{BC}_{3}$ & Individual & $\left(19 \mathrm{~F} 3 \times 10^{\pi} D b\right) \times 20[5]$ & F4 \\
\hline 5 & $\mathrm{BC}_{4}$ & Individual & $\left(19 \mathrm{~F} 4 \times 10^{\pi} D b\right) \times 20[5]$ & $F^{5}$ \\
\hline I4 & $\mathrm{BC}_{13}$ & Individual & $\left(1+\mathrm{d} F 13 \times 30^{\pi} D b\right) \times 20[5]$ & Frt \\
\hline $\begin{array}{r}15 \\
:\end{array}$ & $\mathrm{BC}_{14}$ & Individual & $\left(19 \mathrm{~F} 14 \times 30^{\top} D b\right) \times 20[5]$ & $\begin{array}{l}\text { Fi5 } \\
\vdots\end{array}$ \\
\hline 24 & $\mathrm{BC}_{15}$ & Individual & $\left(19 \mathrm{~F} 23 \times 30^{\prime \prime} D b\right) \times 20[5]$ & F24 \\
\hline 25 & $\mathrm{BC}_{16}$ & Individual & $\left(1 \sigma^{7} \mathrm{~F} 4 \times 39 D b\right) \times 20[5]$ & F25 \\
\hline
\end{tabular}

BC: Backcross.

HC: Hybrid cross.

F: Filial offspring.

$D b$ : Parental strain of Drosophila buzzatii.

$D k o$ : Parental strain of $D$. koepferae.

[] Number of replicates of each series.

Figure 2. Crossbreeding protocol to obtain single-segmental lines: 1) obtaining hybrid offspring; 2) recombinant offspring; 3) multi-segmental offspring in all chromosomes; 4) multiple segmental offspring just in the four chromosome; 5 to 14) selection of single segmental lines exclusively in the four chromosome; 15 to 24) Just single segmental lines with segments of interest; 25) lines in stock.

\subsubsection{Method to obtaining segmental offspring in homozygous state}

The series of crosses to produce homozygous segmental offspring are summarized in figure 3 and described below in a scheme comprising 7 general stages.

According to this crossing scheme, the first obtaining of homozygous segmental offspring corresponding to stage 4 is obtained from the crossing between heterozygous segmental males and females, which for this pair of sibling species $D$. buzzatii-D and Koepferae are fertile (Naveira and Fontdevila 1986) (García-Franco et al, 2020).

1) Backcross $1\left(B_{1}\right)$ and Reciprocal $1\left(B_{1 R}\right)$ : as an example, for obtain new offspring with homozygous (B1/B1) and heterocigous (B1/bu) genotipes, we perform ten individual crosses, using a male of the single segmental line (B1) in mantenance (table 1) with a female from parental strain $D$. buzzati Bu-28 $(D b)$ (table 1). In parallel, 10 individual reciprocal crosses were carried out, using in each crossing 1 female of the B1 single segmental line with a male of the parental strain $D b$. These new offspring were designated as type $\mathrm{F} 2$ (bu/bu, B1/bu) and $\mathrm{F} 2 \mathrm{R}$, (bu/bu, B1/bu) respectively.

2) Isolation of $B 1$ parents of $B C_{1}$ and $B C_{1(R)}$ : for to deplete parent females $B C_{1 R}$ from egg laying, four days after the start of the crosses, the 10 male flies of the segmental line $\mathrm{B} 1$ used as progenitors in the $\mathrm{BC}_{1}$, as well as the 10 female segemental flies $\mathrm{B} 1$ used as progenitors in the $\mathrm{BC}_{1 \mathrm{R}}$, were removed from each of their respective cultures, and each 
of those 20 flies was isolated in a new culture vial, and kept isolated independently until these females stopped laying eggs, 15 to 20 days.

3) Identification genotype parents $B C_{1}$ and $B C_{R 1}$ : To infer the genotype of each of the 10 male $B 1$ parents of the backcrosses, and of the 10 female $B 1$ parents of the reciprocal cultures, kept in isolation. At least 6 larvae from each offspring $F 2$ and $F 2_{R}$ were analyzed cytogenetically for asynapses on polythene chromosomes.

4) Hybrid crooses type $1\left(H_{C_{1}}\right)$. To obtain homozygous hybrid segmental offspring (B1/B1), we made individual crosses using the same parent male of the $\mathrm{BC}_{1}$ cross, previously isolated and identification of its genotype $(\mathrm{B} / \mathrm{bu})$, with the same parent female of the $\mathrm{BC}_{\mathrm{R} 1}$ cross, previously isolated until complete depletion of the laying of eggs (15 to 20 days) and identification of its genotype (B/bu). These new descendants were designated as segmental homozygous type F3 offspring (bu/bu, B1/bu and B1/B1).

5) Backcross $2\left(B_{2}\right)$ and Reciprocal $2\left(B_{R_{2}}\right)$ : we made individual crosses (1 male $X$ 1 female) between the males of the F3 offspring with females of the parental strain Db, and in parallel, we made reciprocal crosses using the females of the F3 offspring with males of the parental strain $\mathrm{Db}$.

These backcrosses represent a dual purpose, 1) they produce, among others, heterozygous segmental cultures with $100 \%$ of the B1/bu flies, which can be used to produce new homozygous offspring, avoiding the isolation processes of the progenitors and their crossing without being virgins, as done in stage 6 .

2) function as test crosses that allow evaluating the fertility of segmental flies (heterozygous and homozygous). These new offspring were designed as type F4 and $\mathrm{F} 4_{\mathrm{R}}$ respectivelly.

6) Hibryd segmental cross $\mathbf{H C}_{1}$ : we made individual type crosses (1 male $X 1$ female), using male and female siblings only of the $F 4_{R}$ heterozygous segmental offspring, from the reciprocal cross $\left(B C_{R 2}\right)$, but we only used flies from $F 4_{R} C$ cultures with $100 \%$ of flies of genotype B1/bu.

We use only the offspring of the reciprocal crosses $\left(B C_{R 2}\right)$ because the segmental parents of homozygous genotype (B1/B1) of these BCR2 crosses are fully fertile while the male parents of homozygous segmental genotype (B1/B1) of the backcrosses (BC2) are affected in your fertility, and therefore do not produce offspring. These new descendants were designated as type F5.

7) Backcross $3\left(B_{3}\right)$ : we carried crosses of individual type (1 male $X 1$ female), using males of homozygous segmental offspring $\mathrm{F} 5$ with parental females $\mathrm{Db}$. In parallel, individual reciprocal crosses were carried out, using in each crossing 1 female of the F5 single segmental line with a male of the parental strain $D b$.

This crossing will allow the second evaluation of the fertility of segmental flies, both heterozygous (B1/bu) and homozygous (B1/B1), according to the number of larvae produced in each of their respective cultures. These new descendants were designated as type F6. 
1)

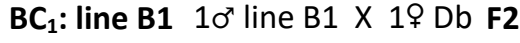

Crosses to obtain segmental homozygous offspring

Generation Crossing Offspring Offspring Crossing Generation

(B1/bu) A (ơbu/buX $9 b u / b u) ~ F 2 A(b u / b u)$

$(\mathrm{bu} / \mathrm{bu}) \quad \mathrm{B}\left(\mathrm{o}^{\circ} \mathrm{B} 1 / \mathrm{bu}, \mathrm{X}\right.$ \%bu/bu)$\rightarrow \mathrm{F} 2 \mathrm{~B}(\mathrm{~B} 1 / \mathrm{bu}, \mathrm{bu} / \mathrm{bu})$

F2 $\mathbf{R}_{\mathrm{R}}$

$19 \mathrm{~B} 1 \mathrm{X} 10^{\pi} \mathrm{Db} \mathrm{BC}_{\mathrm{R} 1}$ : line $\mathrm{B} 1$

2) Isolation of male $\mathrm{C} 1$ parents

3) Genotypes of parents $\mathrm{BC}_{1}$

$\mathrm{HC}_{1}$ : heterozygous

4) segmental parents (B1/bu) from $\mathrm{BC}_{1}$ and $B C_{R 1}$

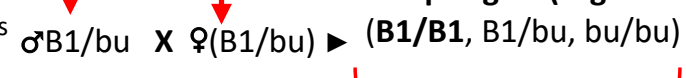

ơ $1 / / b u \quad x \quad \%(B 1 / b u) \vee(B 1 / B 1, B 1 / b u, b u / b u)$

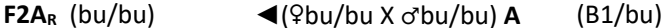

$\mathrm{F}^{2} \mathrm{~B}_{\mathrm{R}}(\mathrm{B} 1 / \mathrm{bu}, \mathrm{bu} / \mathrm{bu}) \varangle\left(\mathrm{QB}^{\mathrm{B}} 1 / \mathrm{bu}, \mathrm{X} \mathrm{o}^{\mathrm{r}} \mathrm{bu} / \mathrm{bu}\right) \mathrm{B} \quad(\mathrm{bu} / \mathrm{bu})$

Isolation of female C1 parents Genotypes of Parents $\mathrm{BCR}_{1}$

5) $\quad \mathrm{BC}_{2}$

of $3 \times$ \% Db $\quad F 4$

$\begin{array}{ccccc}\text { To fertility } & \text { A (bu/bu } & \text { X } & \text { bu/bu) } \\ \text { B (B1/bu } & \text { X } & \text { bu/bu) }\end{array}$ evaluation one

$\begin{array}{lll}\text { B (B1/bu } & X & \mathrm{bu} / \mathrm{bu}) \\ \mathrm{C}(\mathrm{B} 1 / \mathrm{B} 1) & \mathrm{X} & \mathrm{bu}(\mathrm{B} / \mathrm{B} 1 / \mathrm{bu})\end{array}$

6) $\mathrm{HC}_{1}$ : heterozygous ơ $B 1 / \mathrm{bu} \times$ \% $\mathrm{B} 1 / \mathrm{bu}$

Offspring F3 (segmental homozygous 1 BB1) FIRS segmental $(B 1 / \mathrm{bu})$ from $\mathrm{F} 4_{R} C$ )

\begin{tabular}{|c|c|c|}
\hline $\begin{array}{c}F 4_{R} \\
F 4_{R} A(b u / b u)\end{array}$ & $\begin{aligned} \mathbf{F} 3 \quad \mathbf{X} & \mathbf{\sigma}^{7} \mathbf{D b} \\
(\mathrm{bu} / \mathrm{bu}) & \times \mathrm{bu} / \mathrm{bu})\end{aligned}$ & $\mathbf{B C R}_{\mathbf{2}}$ \\
\hline $\mathrm{F}{ }_{\mathrm{R}} \mathrm{B}(\mathrm{B} 1 / \mathrm{bu}, \mathrm{bu} / \mathrm{bu})$ & $\varangle(\mathrm{B} 1 / \mathrm{bu} \times \mathrm{bu} / \mathrm{bu})$ & ertility \\
\hline $\mathrm{F} 4_{\mathrm{R}} \mathrm{C}(\mathrm{B} 1 / \mathrm{bu})$ & $\triangle(\mathrm{B} 1 / \mathrm{B} 1) \times \mathrm{bu} / \mathrm{bu})$ & \\
\hline
\end{tabular}

Offspring F5 (segmental homozygous 2 BB1)

(B1/B1, B1/bu, bu/bu)

$\mathbf{B C}_{3}$
To fertility
evaluation two
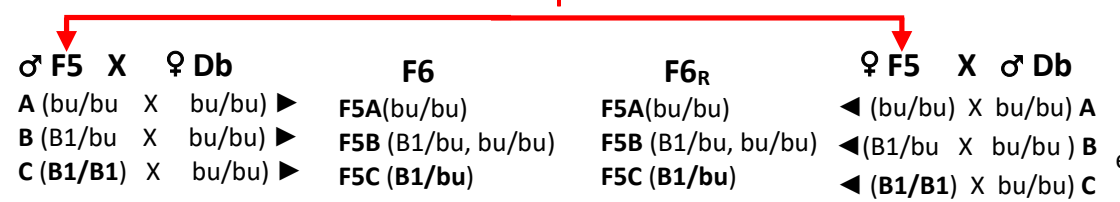

$\mathrm{BCR}_{\mathbf{3}}$ To fertility

BC: Backcross

$\mathrm{BC}_{\mathrm{R}}$ : Reciprocal backcross

HC: Hybrid cross

F: Filial offspring

B1: segmental heterozygous line

BB1: Segmental homozygous offspring

Dbu: Parental strain of Drosophila buzzatii

bu/bu: Genotype of parental Db

B1/bu: Genotype of heterozigous segmental

B1/B1: Genotype of Homozygous segmental

Figure 3. General scheme to obtaining segmental offspring in homozygosity.

\subsubsection{Test crosses}

We made individual test crosses, one male with two females, using males obtained from each homozygous simple segmental offspring (table 3 ) with parental $D$. buzzatii Bu-28 strain $(D b)$ females (table 1$)$.

Parallel to each test cross, we made their respective reciprocal cross, between corresponding females from each homozygous simple segmental offspring (table 3 ) with Bu-28 strain $(D b)$ males (table 1 ). In test crosses the number of adults produced from each cross was counted during 20 days from the first day of emergence. In crosses that failed to produce offspring, we added two new virgin females to confirm male sterility.

Male progenitors are considered fertile when their corresponding crosses produce a number of offspring similar to that of control crosses $(>60)$, they are considered semisterile when their offspring represent only a low percentage of the control's progeny (at least 1 less than 15), finally, they are considered sterile when no offspring are produced. 


\subsubsection{Mapping the smallest homozygous sterility segments}

\subsubsection{In situ hybridization \\ 1.1.1.1 Obtaining BAC clones of Genomics library of Drosophila buzzatti}

The maping and obtantion the genetic sequences, of the small chromosomal fragment of interest (F2-F4), it has been made using the genomics CHORI-BAC 265 (Bacterial artificial chromosome) library of $D$. buzzatii (fig. 6), obtained from the BACPAC Resource Center (BPRC) attached to the Oakland Children's Hospital Research Institute (CHORI).

This genomics BAC library was built by The Research Group on Genomics, Bioinformatics and Evolution of the Autonomous University of Barcelona, based in a physical map of the six chromosomes that make the Drosophila buzzatii genome, for this purpose it assembled the all fragments of the 6 chromosomes in BACs (Gonzalez et al, 2005). The following figure shows the physical map of chromosome 4, where the positions of the respective BAC clones containing each identified chromosomal fragment (figure 4).

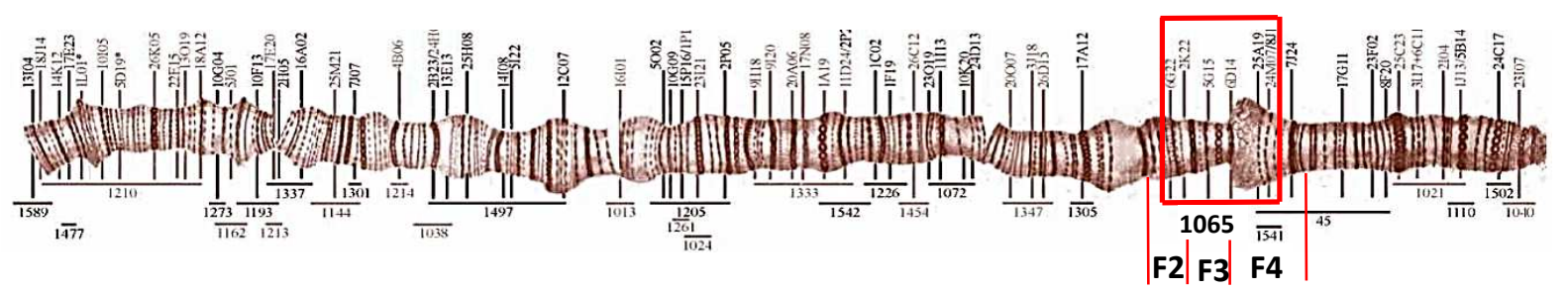

Figure 4. Integrated BAC-based physical map of the Drosophila buzzatii genome. Vertical lines indicate the relative position of the each of the $65 \mathrm{BAC}$ clones label. The 31 contigs to which the hybridized clones belong are represented by short horizontal lies below the chromosomes along with the respective contig number. Into the red rectangle, the position of contig 1065 is indicated where the chromosomal fragments of interest F2, F3, and F4 are located. (Modified image from Gonzalez et al, 2005).

Of the 31 contigs of chromosome four (fig. 4), the contig selected for our research is number 1065, which is made up of 63 BAC clones, each registered with a consecutive number that goes from number 28 to number 90 (table 1).

The chromosomal region encompassing contig 1065 includes the chromosomal segment of interest F2 (F2a-F3d) (Table 3 and Fig. 4). Therefore, in order to map said segment with genetic tags, we decided to acquire 8 clones (41.Z006D14, 47. Z025K20, 53.Z021N13, 59. Z015H23, 64. Z005G15, 76. Z005012, 82. Z002K06 and 86. Z002K22) of the contig 1065 that are distributed along said chromosomal region see table X. Said clones were acquired from the company BACPAC Resource Center (BPRC) attached to the Oakland Children's Hospital Research Institute (CHORI). 
Table 2. Key assigned to each of the 63 BAC clones that make up contig 1065, labeled with consecutive numbers ranging from number 28 to number 90 .

\begin{tabular}{|c|c|c|c|c|c|c|c|}
\hline 28. Z006E21 & 36. Z019M16 & 44. Z012N02 & 52. Z001C10 & 60. Z003M07 & 68. Z024F14 & 76. Z005012 & 84. Z018E16 \\
\hline 29. Z010D13 & 37. Z025C20 & 45. Z021A12 & 53. Z021N13 & 61. Z017K07 & 69. Z001M11 & 77. Z005M13 & 85. Z000G17 \\
\hline 30. Z010C14 & 38. Z022E22 & 46. Z009D02 & 54. Z006L04 & 62. Z026HO9 & 70. Z016A09 & 78. Z024A05 & 86. Z002K22 \\
\hline 31. Z006PO2 & 39. Z009K38 & 47. Z025K20 & 55. Z008M20 & 63. Z002023 & $71 . \mathrm{ZO26L2D}$ & 79. Z012H2O & 87. Z018H13 \\
\hline 32. Z017D13 & 40. Z006C14 & 48. Z005120 & 56. Z010A09 & 64. Z005G15 & 72. Z026P16 & 80. Z004M16 & 88. Z005023 \\
\hline 33. Z017H02 & 41. Z006D14 & 49. Z025K11 & 57. Z0118A20 & 65. Z006M04 & 73. Z003A15 & 81. Z007N14 & 89. Z017P09 \\
\hline 34. Z007CO2 & 42. ZO20E10 & 50. Z006D03 & 58. Z019E05 & 66. Z010A10 & 74. Z018L10 & 82. Z002KO6 & 90. Z008H18 \\
\hline 35. Z009C17 & 43. Z009D01 & 51. Z024C01 & 59. Z015H23 & 67. Z012K04 & 75. Z022B21 & 83. Z008020 & \\
\hline
\end{tabular}

\subsubsection{In situ hybridization using BACs clones as probes}

The DNA of BAC clones was used as an in situ hybridization probe, to confirm the hybridization of these clones in the F2 and F3 (fig. 6) banding region of chromosome 4 of D. Buzzatii, as well as in the species D. Mojavensis, a synmorphic species of the group packed.

The BAC clones were labeled with biotin by the "Nick Translation" method, using the protocol of the commercial kit (BioNick kit, Gibco-BRL).

After labeling the probe, the next steps of the in situ hybridization process consist of preparing the polytechnic chromosomes, prehybridization, hybridization, posthybridization, development and visualization, as described in CarbajalRodriguez, 1999.

The labeled probes hybridized homologously to the polytechnic chromosomes of D. buzzatti, and heterologously to the polytechnic chromosomes of $D$. mojavensis.

Each of the in situ hybridization preparations were observed with an optical microscope and photographs were taken at 40 and 100X.

The in situ hybridization signals have been located and numbered based on the band pattern determined by chromosome 4 of $\mathrm{D}$. repleta.

\section{Results}

\subsection{Evaluation of chromosomal segments in homozygosity}

\subsubsection{Obtaining simple segmental lines in heterozygosity}

The original fly strains, D. koepferae KO-2 (Dko) and D. buzzatii Bu-28 $(\mathrm{Db})$, provided the material to obtain single segmental lines (Table 1) following a strict mating strategy (fig. 2).

According to the scheme of crosses, proposed in figure 2, six simple segmental lines in heterozygous state were obtained, which are described below in table 3.

\subsubsection{Obtaining single segmental lines in homozygosity}

By strictly following the crossing protocol, described in figure 3, it was possible to obtain six segmental lines in the homozygous state (Table 3 ).

Figure 3 describes the crossing scheme that includes up to 27 crosses, which depends on the selection of segments of interest obtained in each new offspring. 
Table 3. Segmental lines obtaining

\begin{tabular}{|c|c|c|c|c|c|}
\hline \multirow{2}{*}{$\begin{array}{c}\text { No } \\
1\end{array}$} & \multicolumn{2}{|c|}{$\begin{array}{l}\text { Heterozogous } \\
\text { single segmental } \\
\text { lines }\end{array}$} & \multicolumn{2}{|c|}{$\begin{array}{l}\text { Homozygous } \\
\text { segmental offsprings }\end{array}$} & \multirow{2}{*}{$\begin{array}{r}\text { Segment size relative } \\
\text { of chromosome } 4(\%) \\
460\end{array}$} \\
\hline & Name & Genotype & Name & Genotype & \\
\hline 2 & F2 & F2/bu & FF2 & F2/ F2 & 6.25 (F2a-F3d) 461 \\
\hline 3 & F3 & F3/bu & FF3 & F3/ F3 & 7.87 ( F3a-F4h ) 462 \\
\hline 4 & B1 & B1/bu & BB1 & B1/ B1 & 10.59 (B1a-B4f )463 \\
\hline 5 & FG & $\mathrm{FG} / \mathrm{bu}$ & FFGG & FG/ FG & 21.87 ( F3a-G5) 464 \\
\hline 6 & D3 & D4/bu & DD4 & D4/ D4 & 9.06 ( D4a-E1h ) 465 \\
\hline 7 & D1 & $\mathrm{D} 1 / \mathrm{bu}$ & DD1 & D1/ D1 & $15.62(\mathrm{D} 1 \mathrm{a}-\mathrm{D} 5 \mathrm{~d})$ \\
\hline
\end{tabular}
obtained from each homozygous simple segmental offspring (table 3) with parental type $\mathrm{Db}$ females (table 1). Parallel to each test cross, we made their respective reciprocal cross, between corresponding females from each homozygous simple segmental offspring (table 3 ) with Db males (table 1). According to the results of the test crosses recorded in table 4, we used three fertility criteria (table 5), based on the total number of larvae present in all the culture flasks that make up each of the different offspring, including the total number of larvae from each offspring of parent females isolated during depletion of egg-laying: Fertile: the presence of at least 60 larvae. Sterile: number zero of larvae. 
Table 4. Result of the number of descendants of each individual test crosses, according to the type of genotype of each parent.

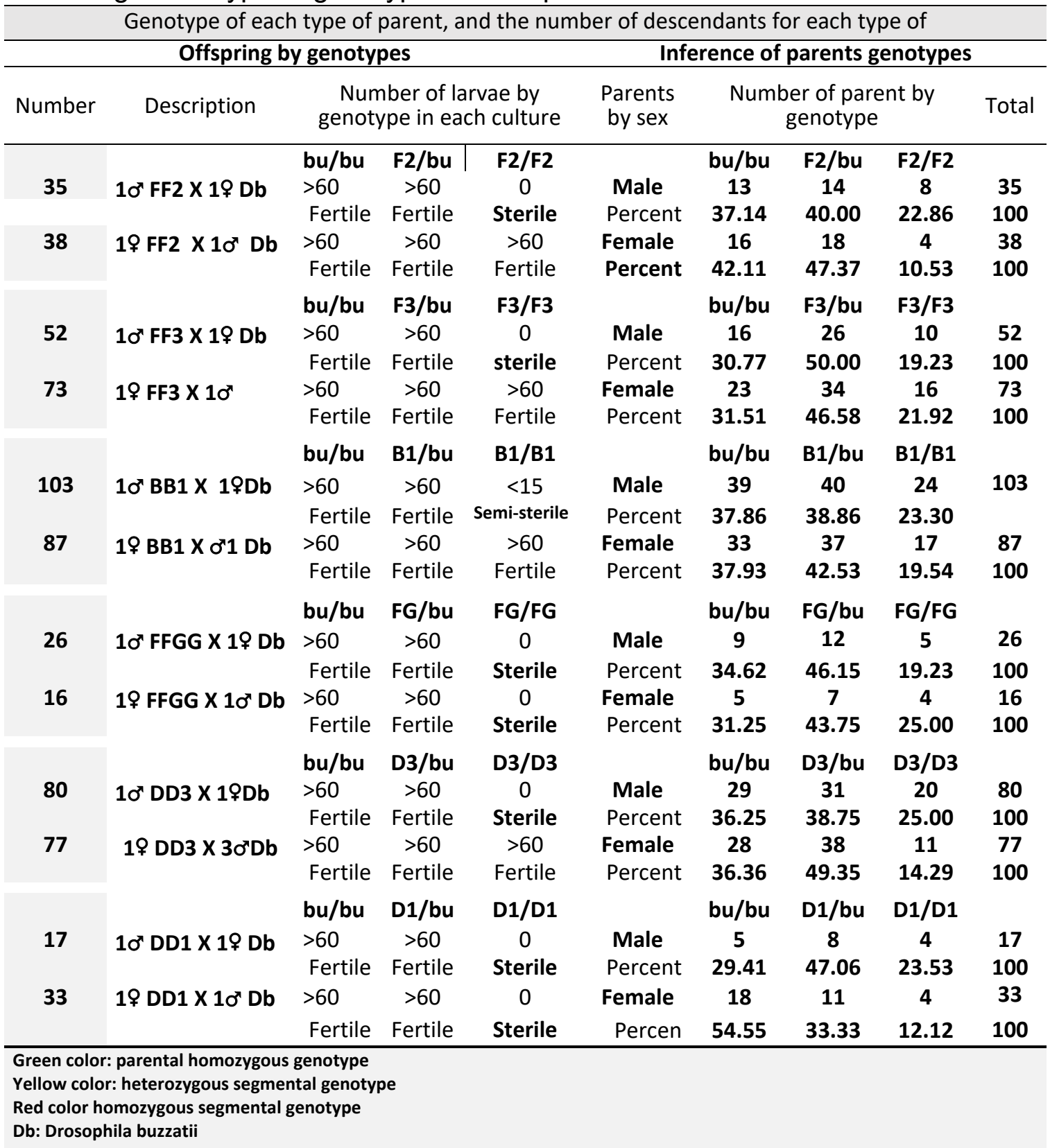

The figure 5 shows offspring according to their introgressive segments (B1, D3, $D 1, F 2, F 3$, and FG), differentiating the numbers of males $(m)$ and females ( $f$ ) organized in their respective genotypes (parental, segmental heterozygous, segmental homozygous).

513 While in figure 6, the data of the total numbers of parents of each offspring are grouped, differentiating the trend of the presence of females and males by the types of genotypes of each offspring. 
Fig. 5

523

524

525

526

527

528

529

530

531

532

533

534

535

536

537

538

539

540

541

542

543

544

545

546

547

548

549

550

551

552

553

554

555

556

557

558

559

560

561

562

563 5.

Fig. 6

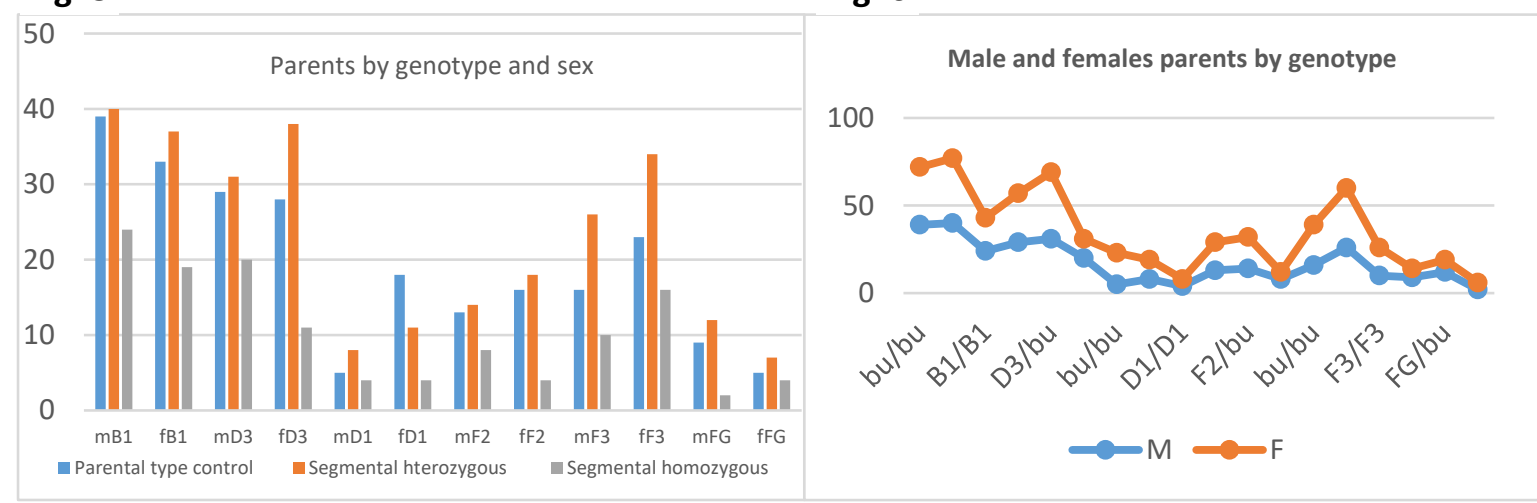

Figure 5. Offsprings grouped according to their introgressive segment (B1, D3, D1, F2, $F 3$ and $F G)$, and their sex $(m=$ male, $f=$ female $)$.

Figure 6. Comparison of the number of male and female parents of each type of offspring (B1, D3, D1, F2, F3, and FG) according to their three respective genotypes, parental, segmental heterozygous, and segmental homozygous.

\subsubsection{Fertility heterozigous and homozygous segments}

From the data obtained in the results of the test crosses

(Table 4) it was possible to observe the effect that each segment produces on the fertility of males and segmental females.

The effect on fertility is shown in three levels: 1) total sterility; 2) semi-sterility and 3 ) fertility, and it turns out to be complex as it depends on several factors: segment size, position on the chromosome, sex and genotype, as shown in Table

Table 5. The fertility of parents according to their segmental genotype and sex

\begin{tabular}{|c|c|c|c|c|}
\hline \multirow[t]{2}{*}{$\begin{array}{l}\text { Segment size relative } \\
\text { of chromosome } 4(\%)\end{array}$} & \multicolumn{2}{|c|}{$\begin{array}{c}\text { Heterozygous } \\
\text { segmental parents }\end{array}$} & \multicolumn{2}{|c|}{$\begin{array}{l}\text { Homozigous segmental } \\
\text { parents }\end{array}$} \\
\hline & Name & Fertility & Name & Fertility \\
\hline 6.25 (F2a-F3d) & $\begin{array}{l}\text { on F2 } \\
\text { @ F2 }\end{array}$ & $\begin{array}{l}\text { Fertile } \\
\text { Fertile }\end{array}$ & $\begin{array}{l}\sigma^{x} F F 2 \\
\text { O FF2 }\end{array}$ & $\begin{array}{l}\text { Sterile } \\
\text { Fertile }\end{array}$ \\
\hline 7.87 (F3a-F4j) & $\begin{array}{l}\text { Ó F3 }^{n} \\
\text { o F3 }\end{array}$ & $\begin{array}{l}\text { Fertile } \\
\text { Fertile }\end{array}$ & $\begin{array}{l}0^{\pi} \mathrm{FF} 3 \\
\text { o FF3 }\end{array}$ & $\begin{array}{l}\text { Sterile } \\
\text { Fertile }\end{array}$ \\
\hline 12.25 (B1a-B4f) & $\begin{array}{l}0^{\top} \mathrm{B} 1 \\
\text { o B1 }\end{array}$ & $\begin{array}{l}\text { Fertile } \\
\text { Fertile }\end{array}$ & $\begin{array}{l}\text { ơ BB1 } \\
\text { 오 BB1 }\end{array}$ & $\begin{array}{l}\text { Semiesterile } \\
\text { Fertile }\end{array}$ \\
\hline 21.87 (F3a-G5c) & $\begin{array}{l}\sigma^{7} \mathrm{FG} \\
\text { ९ FG }\end{array}$ & $\begin{array}{l}\text { Fertile } \\
\text { Fertile }\end{array}$ & $\begin{array}{l}\text { o' FGFG } \\
\text { ९ FGFG }\end{array}$ & $\begin{array}{l}\text { Sterile } \\
\text { Sterile }\end{array}$ \\
\hline 12.25 (D3a-E1h ) & $\begin{array}{l}\text { ơ D3 } \\
\text { ᄋ D3 }\end{array}$ & $\begin{array}{l}\text { Fertile } \\
\text { Fertile }\end{array}$ & $\begin{array}{l}0^{7} \mathrm{DD} 3 \\
\text { @ DD3 }\end{array}$ & $\begin{array}{l}\text { Sterile } \\
\text { Fertile }\end{array}$ \\
\hline 15.62(D1a-D5d) & $\begin{array}{l}0^{x} \mathrm{D} 1 \\
\text { ᄋ D1 }\end{array}$ & $\begin{array}{l}\text { Fertile } \\
\text { Fertile }\end{array}$ & $\begin{array}{l}\text { ó DD1 } \\
\text { @ DD1 }\end{array}$ & $\begin{array}{l}\text { Sterile } \\
\text { Sterile }\end{array}$ \\
\hline
\end{tabular}

In Figure 7 the twelve segments (Table 3 ) typical of this study (6 analyzed in heterozygosity and 6 analyzed in homozygosis) are represented in a schematized way, the segments of the studies by Garcia-Franco et al 2020 are 
564 also found, the de Naveira and Fontdevila 1986, 1991a, 1991b, as well as the 565 segments proposed by I. Marin, 1996.

566 All those chromosomal segments mentioned above, with the exception of the 6 567 segments analyzed in this work, were evaluated in their respective publications, 568 only in the heterozygous state for their ability to possess hybrid sterility genes.

569 In the following diagram, each of these segments are located in their 570 corresponding position within chromosome 4 of the full group and according to 571 their relative size in percentage in relation to the total size of the chromosome 4.

A
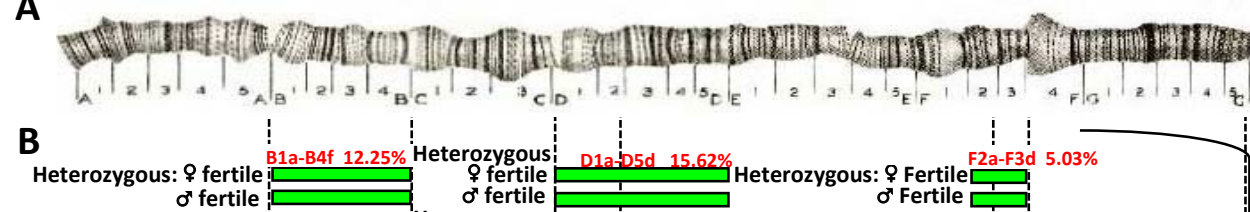

Homozygous: क fertile
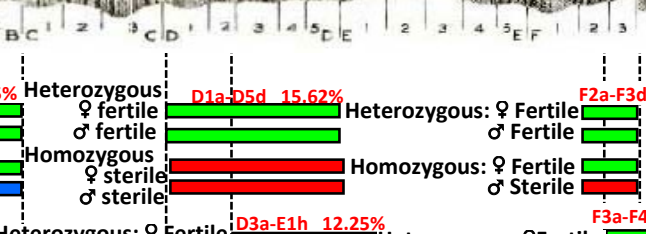
o' semiesterile

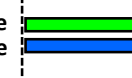

!
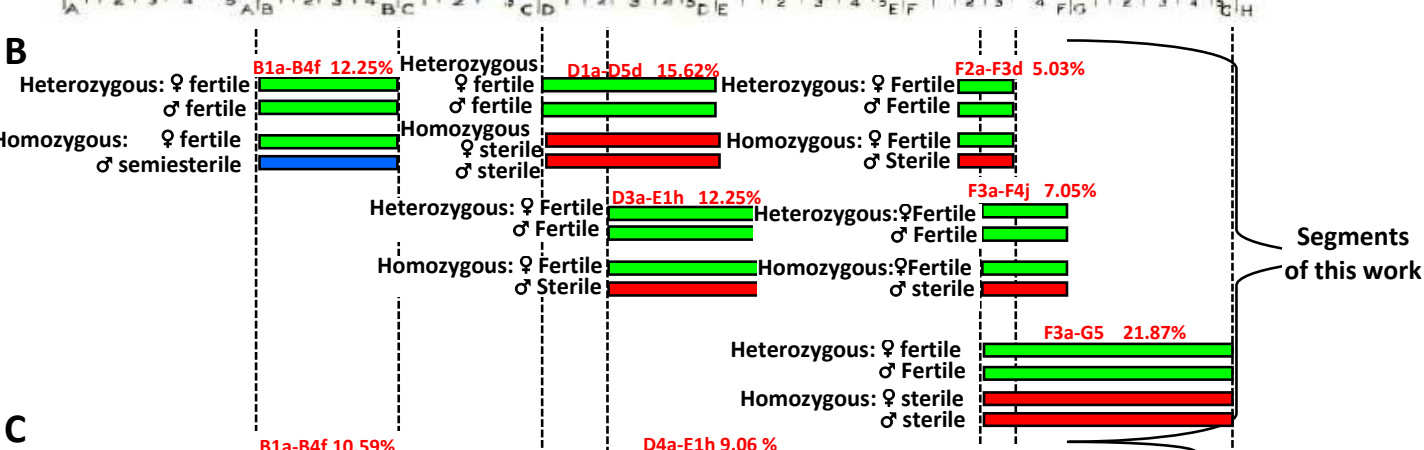

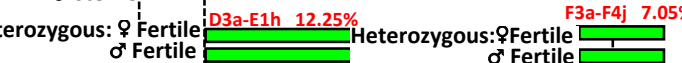

Heterozygous:PFertile
of Fertile

sterile

Segments

C

Heterozygous: \% Fertile
ơ Fertile

Heterozygous: 9 Fertile

B1a-B4f $10.59 \%$

。 a-B4f $10.59 \%$

s.

o' Fertile : 9 sterile

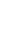


The name and size of each segment can be seen at the top of its respective segment.

\subsection{Mapping the smallest homozygous sterility segments 3.2.1 BAC clones of $D$. buzatii}

Eight BACS clones out of a total of 63 that make up contig 1065 (Tables 2 and 6 ), was acquired from the Oakland Children's Hospital Research Institute (CHORI).

The Contig 1065 encompasses almost the entire F2 toF4 region (Fig. 4) of chromosome 4 of the physical map of $D$. buzzatii.

The eight clones were selected to demonstrate, by in situ hybridization, the correspondence of Contig 1065 in the F2 to F4 region.

Table 6. Selected clones of Contigo 1065

\begin{tabular}{|l|l|}
\hline \multicolumn{2}{|l|}{ Clones de Conti 1065 } \\
\hline Número & Clave \\
\hline 41 & Z006D14 \\
\hline 47 & Z025K20 \\
\hline 53 & Z021N13 \\
\hline 59 & Z015H23 \\
\hline 64 & Z005G15 \\
\hline 76 & Z005012 \\
\hline 82 & Z002K06 \\
\hline 86 & Z002K22 \\
\hline
\end{tabular}

608

609

610

611

612

613

614

615

616

617

618

619

620

621

622

623

624

625

626

627

628

629

\subsubsection{In situ hybridization}

According to the proposed cytogenetic relationships between $D$. buzzatii and $D$. mojavensis, the two species are close enough to maintain a high homosequence ratio, especially for chromosome 4 because tothe high colinearity (Guillen et al, 2018) so we performed in situ hybridizations with the same probes for both species.

In situ hybridizations were carried out using each of the 8 BAC clones as probes, marking them with biotin. The probes were hybridized in polythene chromosomes of D. buzzatii and D. mojavensis, respectively. The eight probes performed by in situ hybridizations were positive. In figure 7, 4 of them are shown.

A

B

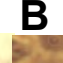

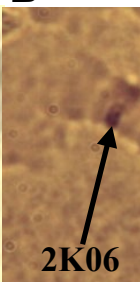

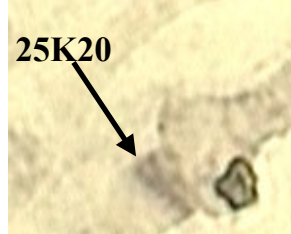

C

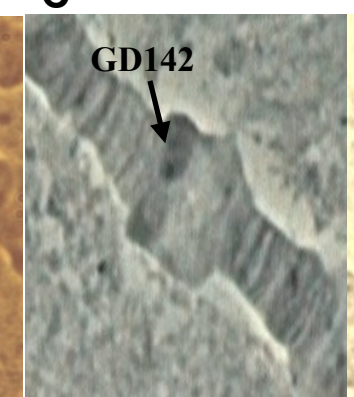

D

Figure 8. Positive signals from in situ hybridizations. A) and B) positive signals from probes $25 \mathrm{~K} 20$ and $2 \mathrm{~K} 06$ in D. buzzatii. C) GD142 probe in D. buzzatii. D) GD142 probe in D. mojavensis.

The eight probes prepared from the BACs clones hybridized in the precise position expected for both $D$. buzzatii and $D$. mojavensis, in both species within 
the F2 to F4 region, according to the physical map of chromosome 4, as outlined in figure 98.

\author{
D. buzzatii
}
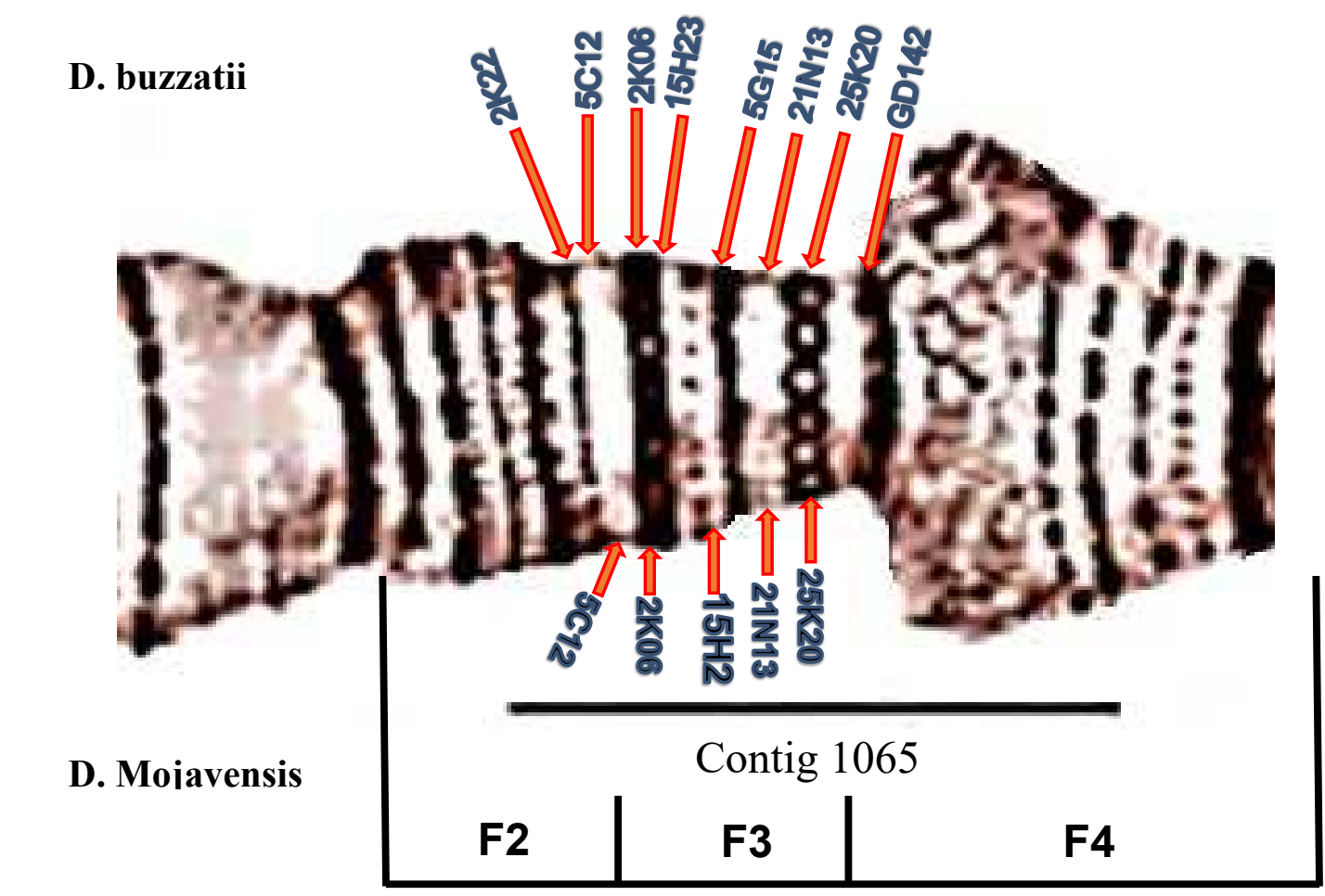

Figure 9. In situ hybridizations of 8 BACs clones in D. buzzatii and D. mojavensis. The arrows in the upper part corresponding to the hybridizations in polygenic chromosomes of D. buzzatii, and the arrows in the lower part those of D. mojavensis. Each arrow indicates the position of the hybridization signal corresponding to the respective probe according to the indicated key. The black line under the chromosome image represents the full length of contig 1065. The 3 regions F2, F3 and F2 of chromosome 4 are indicated.

\title{
3.3 Bioinformatic analysis \\ 3.3.1 BLAST Procedure
}

Using the gene browser of the D. buzzatii from the Drosophila buzzatii Genome Projet (http://dbuz.uab.cat) we obtained the nucleotide sequence of contig 1065 and access to the genomic map of $D$. buzzatii to know the genes and genetic accessories annotated that are associated with contig 1065.

The complete sequence of contig 1065 has 4233 nucleotides, it is found within the scaffold2 between positions 308944 to 313176 of the genomic map of Drosophila buzzatii (Fig. 10A).

BLAST 1: We performed a Standard Nucleotid BLAST (Basic Local Alignment Search) process with the BLAST-suite software from the National Center for Biotechnology Information (NCBI), hosted on the GenBank website, using the complete nucleotide sequence of the Contig 1065 of $D$. buzzatii comparing it with the sequences annotated for the species of the family Drosophilidae (taxid: 7214) housed in GenBank (Thurmond et al., 2020).

The BLAST procedure with the megablast algorithm for highly similar sequences, identified the region with the highest homology:

Is a zone of 3428 nucleotides in the $R$ arm of chromosomal 3 of Drosophila melanogaster, with 11 different segments homologous, which is located within the trivet 
gene in to contig 1065, between the nucleotide region Dmel-chr3R 28074717-28065867 (fig10B and 11C).

We performed a second BLAST procedure, between the nucleotide sequence of contig 1065 and the nucleotide sequences of the Drosophila genus species housed in the FlyBase Gene Bank. We identified an area of 3704 nucleotides with 20 different homologous segments, which is located within the splicing factor (D. moj_GI22766) in to contig 1065belonging to Drosophila mojavensis in the genomic region 19163410-19 167114 of scaffold 6540 (Fig 10C and 11B).

A) Drosophila buzzatii Genome Project (DBGP)

D. buzzatii-chr_4. Scaffold2, contig 01065, length $=4233$ nt (308944 a 313176)

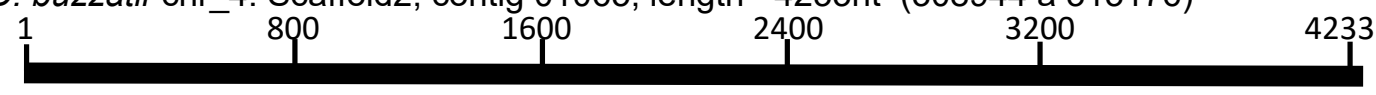

B) GenBAnK. BLAST. Job title: Contig 01065 length $=4233$

Optimize for Highly similar sequences (megablast)

D. melanogaster-chr3R (28074717-28065867)

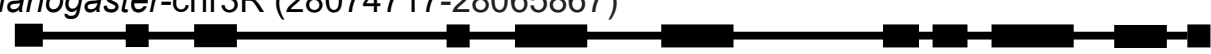

C) FlyBase. BLAST. Contig01065 length $=4233 \mathrm{bp}$

BLAST 3 Dmoi scaffold 6540 (19163410-19 167114)

Figure 10. Schematic representation of the homologous sequences identified by the Blast procedure, between Contig 1065 and the nucleotide sequences of the species of the genus Drosophila. A) Contig 1065 of 4233 nucleotides in total length. B) Eleven homologous segments between the $D$. melanogaster sequences and the 1065 contig. C) Twenty homologous segments between the $D$. mojavensis sequences and the 1065 contig.

Each rectangle indicates a homologous segment between the respective Drosophila species with the 1065 contig of $D$. buzzatii.

\subsubsection{Genes and genetic accessories associated with contig 1065}

According to the genomic map of $D$. buzzatii (Drosophila buzzatti genome project http://dbuz.uab.cat) the nucleotide sequence of contig 1065 is part of the predicted gene Dbuzlg1313.t1, which is located at position 294049-325520 and showing the presence of an orthologous gene (fig 11A).

The orthologous gene is called DmojlG122766 and is described as the splicing factor, proline- and glutamine-rich (splicing factor, proline- and glutamine-rich) whose genomic position includes nucleotides 19150747-19219807 in the mojavensis genomic map (GenBanK_LOC6573952) (fig. 11B).

Dmoj_GI122766 gene is also orthologous with Trivet gene of $D$. melanogaster, identified as regulation of alternative mRNA splicing via spliceosome, being its position within the genomic map of D. elanogaster 28026615-28090740 (Genbank_Trivet) and in that genomic region it is found locally associated with a natural mobile element (DmellI \{\} 1441), and 4 other mobile element insertion sequences (1) Mi $\{E T 1\}$ trvMB02668 , 2) PBac $\{W H\}$ trvf00299, 3) Mi $\{E T 1\}$ trvMB04653 and Mi \{ET1\} MB00028) (fig.11C).

On the other hand, and interestingly, in the genomic map of $D$. mojavensis the DmojlG122766 gene in position 19150747-19219807 is located adjacent to the Dmoj_Gl22767 gene, which is a gene characterized in GenBank as nucleosin TIA-1 (nucleolysin TIA-1) located at position 18977154-19072104 (LOC6573951), and is orthologous to the D. mel_CG34354 gene of D. melanogaster, and is characterized as an RNA-binding domain. This CG34354 gene, described as Trivet's paralogue, in the genomic map of $D$. melanogaster $D$. melanogaster is associated with a natural mobile element (Dmellmdg1 \{\} 1442), and 6 insertion sequences of mobile elements numbered from 1 to 6 ; (1 DmellMi \{ET1\} CG34354MB01203, 2) DmellMi \{ET1\} CG34354MB01203, 
3) Dmel \Mi \{MIC\} CG34354MI03256, 4) DmellMi \{ET1\} CG34354MB07567, 5) Dmel \ Mi $\{$ MIC $\}$ CG34354MI13643, 6) Dmel \P \{GSV2\} GS51766 (Fig. 11C).

$0.0 \mathrm{~K} 10 \mathrm{k}$ 20k 30k 40k 50k 60k 70k 80k 90k 100k 110k 120k 130k 140k 150k 160k 170k 180k 190k 200k 210k 220k 230k 240k 250k 260k 270k 280k
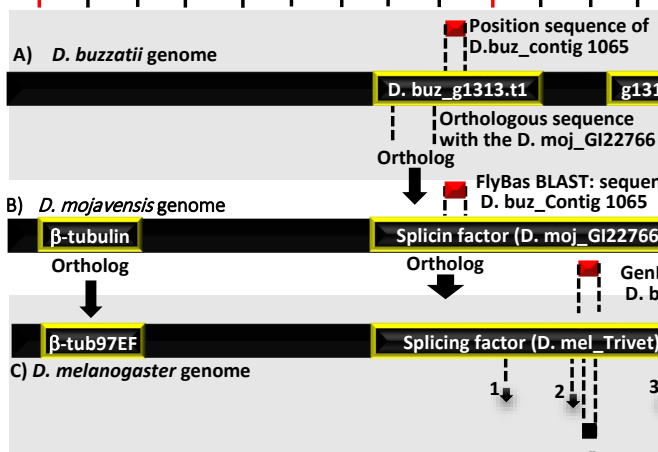
$\overline{9886 \mathrm{Dmel}} \frac{98 \mathrm{~B} 7 \mathrm{Dmel}}{98 \mathrm{~B} 8 \mathrm{Dmel}}$

ET_K\}1441
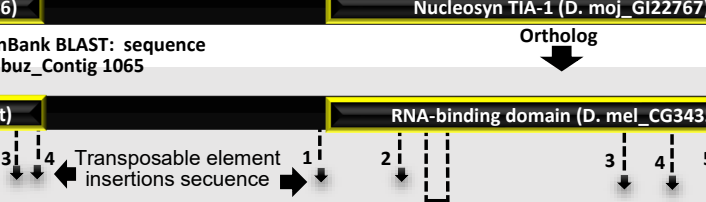
Ortholog
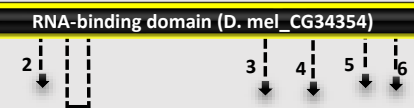
98

Figure 11. Genetic regions of the genomic maps. A) D. buzzatii; B) D. Mojavensis and C) D. melanogaster, which are associated with the nucleotide sequence of contig 1065 of $D$. buzzatii. The numerical scale in kilobases at the top indicates only the relative distance of each genetic element on its respective genomic map.

The long black lines indicate the genomic region represented, respective of each species. Black rectangles with yellow margins represent the position of each of the indicated genes.

The small rectangles in red color indicate the positions of the homologous sequences between the 1065 contig with the genome of $D$. buzzatii, D. mojavensis and $D$. melanogaster respectively.

The smaller black rectangles at the bottom locate the natural transposable elements of each gene. The numbers 1 to 6 indicate the presence of the mobile element insertion sequences and the lower black lines represent the regions of the cytogenetic bands 98B6 to $98 \mathrm{C} 2$ of the cytogenetic map of D. melanogaster.

\section{DISCUTION}

Previous studies on reproductive isolation between Drosophila buzzatti and Drosophila koepferae were always carried out with introgressions of heterozygous chromosomal segments (Naveira and Fontdevila 1986) (García Franco et al., 2020) and it was never possible to identify individual genes with strong effect, although it was able to demonstrate the effect of sterility in segmental males due to a threshold size when it was possible to introgress a segment with a minimum total size of $31.15 \%$ with respect to the total size of chromosome 4, either individually, in pairs or trios, or, both in cis action and in trans action, but always in heterozygosity, as shown in Figure 7. Therefore, with this methodology for obtaining homozygous introgressing segments, we now have the ability to produce an enormous range of homozygous introgressive segments and be able to evaluate them, not only for these species but for any group of species that present $e$ fertile segmental males in heterozygosis, and importantly, being able to isolate groups of small individual introgressing segments that could be associated with one or a few hybrid sterility factors and be able to differentiate them from those that act epistatically between genes of different loci.

Obtaining 6 introgressive segments in a heterozygous state (fig. 2) with sizes between $6.25 \%$ the smallest and up to $21.87 \%$ the largest (table 3 ), did not produce any effect of sterility in males or segmental females, such as can be seen in table 5 .

But when they were obtained in a homozygous state (figure 3), they now showed that the 6 segments were capable of producing some effect on sterility (table 5), and not only in males but also in segmental females, depending not only on size of the segment, but of its position within chromosome 4. 
In such a way that, the segments of size $6.25 \%$ (F2a-F3d), 7.87\% (F3a-F4j) and $12.25 \%$ (B1a-B4f) located in the $F$ region and the first two and the third in the $D$ region respectively, they were able to produce sterility in segmental males, although in females, its effect was null. While an intermediate size segment $(12.25 \%)$ located in region B produced semi-sterility in males and no effect in segmental females. Unlike the larger segments, $15.62 \%$ (D1a-D5d) and $21.87 \%$ (F3a-G5c), located in the D and F regions respectively, produced sterility in both females and segmental males.

The above indicates the potential presence of a single strong effect factor with the capacity to produce sterility in segmental males carrying the smallest segments F2a-F3d $(6.25 \%)$ and $\mathrm{F} 3 a-F 4 j(7.87 \%)$, although we cannot rule out the possibility of epistasis with other factors.

In addition, three of the segments (B4c-B4e, D5b-E1a and F2a-F3d) (fig 7D), proposed by Marin, 1996 as having epistatic sterility factors, coincide in their position with segments of this work (B1a-B4f, D3a-E1h, F2a-F3d) (Fig. 7B), especially the region that overlaps between segments $\mathrm{F} 2 \mathrm{a}-\mathrm{F} 3 \mathrm{~d}$ and $\mathrm{F} 3 \mathrm{a}-\mathrm{F} 4 \mathrm{j}$, and that indicates an even smaller region ( $\mathrm{F} 3 \mathrm{a}-\mathrm{F} 3 \mathrm{e})$, with the possibility of the presence of a single strong effect factor, which could act independently, the same happens with the overlapping segment between the segments D1a-D5d and D3a-E1h, the small region D1a-D2e.

Also in relation to size, it is very revealing that the segments when they are small only affect segmental males but when a larger region is considered (F3a-G5) they also affect segmental females, which suggests additive or epistatic action. . Including the different effect observed between two segments of the same size (12.25\%), B1a-B4f and D3a$\mathrm{E} 1 \mathrm{~h}$, the first produce semi-sterility and the second sterility, in males (table 5 and figure 7).

On the other hand, the effect observed in the 6 segments regardless of their position within chromosome 4 indicates the presence of factors distributed throughout the entire chromosomes, which supports Naveira's original proposal (Naveira and Fontdevila, 1986) (Naveira et al., 1986) Naveira and Fontdevila, 1991a) (Naveira and Fontdevila $1991 \mathrm{~b}$ ) in the extensive study that he has carried out with this pair of species, where he has demonstrated the effect of segment size throughout the genome.

Although each of the 6 homozygous segments is of comprehensive interest, we decided to focus on the smaller F2a-F3d and F3a-F4j segments (Table 5) that produce sterility in segmental males. Thanks to the physical map of Drosophila buzzatii based on BACs (Gonzalez et al, 2005), we managed to obtain (from the BACPAC Resource Center $\mathrm{CHORI}$ ) the 8 clones, which are located along the small region F2 to F4 of the chromosm map of D. buzzatii (Figure 4 and Table 2, and successfully hybridize each one of them when using them as probes, in the polygenic chromosomes of $D$. buzzatii and $D$. mojavensis (Figures 8 and 9). With which we show that we are within region F2 to F4.

The bioinformatic study, using BLAST processes and identification of genes and annotated genetic structures of the genomes of D. buzzatii (Drosophila buzzatii Genome Projet), D. mojavensis and D. melanogaster (GenBank and Flybase) allowed us to focus the genetic region of $D$. . buzzatii where the contig 1065 sequence is located within the genome of $D$. buzzatii, turning out to be an interesting region, which although in $D$. buzzatti only shows the presence of two predicted genes, g1313.t1 and g1314.t1, (figure $11 \mathrm{~A}$ ). An orthologous gene of the g1313.t1 gene is also shown, which is located in the genome of D. mojavensis and is known as the Dmoj_Gl22766 gene (Fig. B), which has a biological function of participating in the regulation of splicing processes, in turn. Once this gene GI22766 is orthologous with its counterpart in D. melanogaster known as the Trivet gene (Fig. $11 \mathrm{C}$ ) and which has the same biological function. And these regions, both of D. mojavensis and D. melanogaster, represent regions of high genetic mobility, since at least one mobile element and several insertion sequences of mobile elements are associated with these genes (Fig. 11C), and Interestingly, this entire region is located in a space of only 5 cytogenetic bands of the D. melanogaster genome (Fig. $11 \mathrm{C}$ ). And although the diagram in figure 11 only shows a small region of 280 kilobases, when comparing the regions of adjacent to this region it is possible to identify that the groups 
of synthenic genes are maintained with great consistency between $D$. buzzatti and $D$. melanogaster, and they are also maintained, although to a lesser degree, between $D$. mojavensis and D. Buzzatii.

With all of the above, we conclude that the method to obtain homozygous segmental offspring allows for the first time homozygous introgressions with the ability to independently evaluate introgressive segments, or it is possible to extend the scheme for joint-segment analysis.

None of the 6 introgressing segments in heterozygosis produce effects on sterility. In contrast, in the homozygous state, the 6 segments produce different effects on sterility, when they are small segments (5 to $7 \%$ ) they produce effects on the sterility of segmental males, and when they are larger $(12.25 \%$ to $21.87 \%)$ they produce semi-sterility. or sterility in segmental males or sterility in both females and segmental males. That the sterility factors extend throughout the entire chromosome 4 considering that they have evaluated in percentage $57 \%$ with the 6 homozygous segments. And that the genes g.1314.t1 and g1313.t1 of D. buzzatti, both still in a state of prediction, represent being candidate genes involved in the reproductive isolation of these symmorphic species.

Although under the classical Bateson-Dobzhansky-Muller model, we are observing changes in at least two genes, due to the differential interaction and in size and position of large segments or in different positions of the genome, now we also observe the possibility of the action of a single allele with strong effects on non-heterozygous sterility and without epistasis to other genes, even considering the decreased aptitude of that allele that makes it difficult to fix this allele in the population, therefore, it would then be necessary to consider both possibilities, appealing to both models and using new strategies to understand the relationship between both possibilities, especially in small populations and in high-mobility genes. On the other hand, the dominance models show us an additional possibility to address the situations revealed in this work, although it is necessary to know the type of dominance of the allele in question under the current models in relation to the genotypic and phenotypic values and their position within the metabolic flow, and additionally it is relevant to consider the type of inheritance especially if it turns out to be of maternal origin. Finally, It is now feasible to study allele fixation processes as one of the fundamental processes of the hybrid sterility effect.

\section{REFERENCES}

Barbash A. D., Awadalla P. and Tarone M. A. 2004 Functional Divergence Caused by Ancient Positive Selection of a Drosophila Hybrid incompatibility Locus. PLoS Biol. 2, 0839. https://doi.org/10.1371/journal.pbio.0020142.

Barbash A. D., Roote J. and Ashburner M. 2000 The Drosophila melanogaster Hybrid male rescue Gene Causes Inviability in Male and Female Species Hybrids. Genetics 154, 1747-1771.

Blackman B. K. Specition genes 2016. Encyclopedia of Evolutionary Biology, Volume 4, pp. 166-175. doi:10.1016/B978-0-12-800049-6.00066-4.

Brill E., Kang L., Michalak K. and Price D. K. 2016 Hybrid sterility and evolution in Hawaiian Drosophila: differential gene and allele-specific expression analysis of backcross males. Heredity 117, 100-108.

Carbajal Rodriguez Antonio. 1999. Base genética de la inviabilidad en híbridos de las especies gemelas Drosophila koepferae y Drosophila Bizzatii. Universidade da Coruña. Teses de doutoramento. Departamento de Bioloxía Celular e Molecular. ISBN 978-84-692-8225-0 http://hdl.handle.net/2183/5617 (http://hdl.handle.net/2183/5617).

Civetta Alberto and Gaudreau Chelsea. 2015 Hybrid male sterility between Drosophila willistoni species is caused by male failure to transfer sperm during copulation. BMC Evol Biol. 15, 2-8.

Cooper Jacob C., and Phadnis, Nitin. 2016 A genomic approach to identify hybrid incompatibility genes. Fly. 10, 142-148. 
881 Coyne Jerry A. and Orr Allen. 1998. The evolutionary genetics of speciation. Phil. Trans.

R. Soc. Lond. B. 353, 287-305. DOI: 10.1098/rstb.1998.0210.

Drosophila 12 Genomes Consortium. 2007. Evolution of genes and genomes on the Drosophila phylogeny. Vol 450|8 November 2007| doi:10.1038/nature06341.

Durando M. Celeste., Baker H. Richard., Etges J. William., Heed B. Williams., Wasserman Marvin and DeSalle Rob. 2000 Phylogenetic analysis of Replete species group of the genus Drosophila Using Multiple sources of characters. Mol. Phylogenet. Evol. 16, 296-307.

Feller AF, Haesler MP, Peichel CL, Seehausen O. 2020 Genetic architecture of a key reproductive isolation trait differs between sympatric and non-sympatric sister species of Lake Victoria cichlids. Proc. R. Soc. B 287: 20200270. http://dx.doi.org/10.1098/rspb.2020.0270

Fontdevila A., Pla, C., Hasson, E., Wasserman, M., Sanchez, A., Naveira, H., and Ruiz A. 1988 Drosophila koepferae: A new number of the Drosophila serido (Diptera: Drosophilidea) superspecies taxon. Ann. Entomol. Soc. Am. 81, 380-385.

Fontdevila A., Ruiz, A., Ocaña, J., and Alonso, G. 1982. The evolutionary history of $D$. buzzatii. II. How much has chromosomal polymorphism changed in colonization? Evol. 36, 843-851.

García-Franco Francisco, Barandica-Cañon Lilian Milena, Arandia-Barrios Jannitza, Galindo-Pérez Ezel Jacome, Binnqüist Cervantes Sven Gilberto, Martínez-García Martha and Chávez-Sandoval Blanca Estela. 2020. Evaluation of Drosophila chromosomal segments proposed by means of simulations of possessing hybrid sterility genes from reproductive isolation.

Guillén Yolanda; Casillas Sònia; Ruiz Alfredo. 2018. Genome-Wide Patterns of Sequence Divergence of Protein-Coding Genes Between Drosophila buzzatii and D. mojavensis. The Journal of heredity 110(1) DOI: 10.1093/jhered/esy041.

Gomes Suzanne and Civetta, Albert. 2015 Hybrid male sterility and genome wide misexpression of male reproductive proteases. Sci. Rep. 5, 11976. doi: 10.1038/srep11976.

Gonzalez J, Nefedov M., Bosdet I, Casals F, Calvete O, Delprat A, Shin H, Chiu R, Mathewson C, Wyle N, Hoskins R, Schein J. E., de Jong P, Ruiz A. 2005. A BAC-based physical map of the Drosophila buzzatii genome. Genome Research. 15: 885-892.

Gregorius Hans-Rolf. 1992. A single-locus model of speciation. Acta Biotheore 40: 313319. https://doi.org/10.1007/BF00046330

Henderson Daryl S. 2004 Drosophila cytogenetics protocols. Methods in Molecular Biology. Humana Press (Clifton, N.J.), V. 247.

Hurtado Juan, Almeida Francisca, Revale Santiago, Hasson Esteban. 2019 Revised phylogenetic relationships within the Drosophila buzzatii species cluster (Diptera: Drosophilidae: Drosophila repleta group) using genomic data. Arthropod systematics \& Phylogeny. 77 (2): 239-250. DOI: 10.26049/ASP77-2-2019-03

Laayouni H., Santos M. and Fontdevila, A. 2000 Toward a physical map of Drosophila buzzatii: Use of randomly amplified polymorphic DNA polymorphism and sequencedtagged site landmarks. Genetics. 156, 1797-1816.

Lindsley D. I. C Tokuyasu K. T. 1980 Spermatogenesis. In Genetics and Biology of Drosophila, 2nd edn (ed. M. Ashburner and T. R. Wright), pp. 225-294. New York: Academic Press.

Liu B-H. 1998 Statistical Genomics: Linkage, Mapping, and QTL Analysis. CRC Press. Boca Ratón, New York.

Lynch M. and Walsh B. 1998 Genetics and Analysis of Quantitative Traits. Sinauer Associates. U.S.

Machado, L. P. B., Madi-Ravazzi, L., Tadei, W. J. 2006. Reproductive relationships and degree of synapsis in the polytene chromosomes of the Drosophila buzzatii species cluster. Braz. J. Biol. vol.66 no.1b. https://doi.org/10.1590/S151969842006000200010.

Mallet James. 1995 A species definition for the modern synthesis. TREE. 10, 294-299. 
Manfrin, M. H., R. O. A. de Brito, and Sene F. M 2001. Systematics and Evolution of the Drosophila buzzatii (Diptera: Drosophilidae) Cluster Using mtDNA. Annals of the Entomological Society of America. vol. 94, no. 3 333-346

Manzano-Winkler Brenda. His Alexander J., Aarons, Emily K., Noor, Mohamed A. F. 2017. Reproductive interference by male Drosophila subobscura on female $D$. persimilis: A laboratory experiment. Ecol Evol. 7, 2268-2272.

Marín I. 1996 Genetic architecture of autosome-mediated hybrid male sterility in Drosophila. Genetics. 142, 1169-1180.

McKee Bruce D., Yan Rihui and Tsai Jui-He. 2012 Meiosis in male Drosophila. Spermatogenesis. 2, 167-184.

Michalak Pawel., Noor, A. F. Moamhed. 2004. Association of misexpression with sterility in hybrids of Drosophila simulans and D. mauritania. J. Mol. Evol. 59, 277-282

Mueller U.G. and LaReesa W. L. 1999 AFLP genotyping and fingerprinting. TREE. 14, 389-394.

Naveira H. and Fontdevila, A. 1986. The evolutionary history of Drosophila buzzatii. XII. The genetic basis of sterility in hybrids between Drosophila buzzatii and its sibling $D$. serido from Argentina. Genetics. 114, 841-857.

Naveira H., Pla C., \& Fontdevila. 1986. The evolutionary history of Drosophila buzzatii $\mathrm{XI}$. A new method for cytogenetic localization based on asynapsis of polytene chromosomes in interspecific hybrids of Drosophila. Genetica 71: 199-212.

Naveira H., and Fontdevila A. 1991a. The evolutionary history of $D$. buzzatii. XXII. Chromosomal and genic sterility in male hybrids of Drosophila buuatii and Drosophila koepferae. Heredity. 66, 233-239.

Naveira H., and Fontdevila A. 1991b. The evolutionary history of Drosophila buzzatii. $\mathrm{XXI}$. Cumulative action of multiple sterility factors on spermatogenesis in hybrids of $D$. buzzatii and D. koepferae. Heredity. 67, 57-72.

Naveira H., E. Hauschteck-Jungen and Fontdevila A. 1984 Spermiogenesis of inversion heterozygotes in backcross hybrids between Drosophila buzzatii and D. serido. Genetica. 6, 205-214.

Naveira H., E. Hauschteck-Jungen and Fontdevila. A. 1989 Evolutionary history of Drosophila buzzatii. XV. Meiosis of inversion heterozygotes in backcross hybrids between $D$. buzzatii and its sibling $D$. koepferae. Genome. 32, 262-270.

Naveira H., Pla C. and Fontdevila A. 1986. The evolutionary history of Drosophila buzzatii. XI. A new method for cytogenetic localization based on asynapsis of polytene chromosome in interspecific hybrids of Drosophila. Genetica. 71, 199-212 DOI: 10.1007/BF00057693.

Nei Masatoshi and Jianzhi Zhang. 1998 Molecular origin of species. Science Vol. 282, Issue 5393, pp. 1428-1429. DOI: 10.1126/science.282.5393.1428.

Noor A. F. Moahamed., Grams L. Katherine., Berticci A. Lisa., Almendarez Yvette., Reiland Jane., Smith Kelley R. 2001 The genetics of reproductive isolation and the potential for gene exchange between Drosophila pseudoscura and the $D$. persimilis via backcross hybrid males. Evolution, 55, 512-52.

Nosil Patrik and Schluter Dolph. 2011. The genes underlying the process of speciation. Trends in Ecology and Evolution April 2011, Vol. 26, No. 4. DOI: 10.1016/j.tree.2011.01.001.

Oliveira Deodoro C.S.G., Almeida Francisca C., O'Grady Patrick M., Armella Miguel A., Rob DeSalle, Etges William J. 2012. Monophyly, divergence times, and evolution of host plant use inferred from a revised phylogeny of the Drosophila repleta species group. Molecular Phylogenetics and Evolution 64 533-544. doi: 10.1016/j.ympev.2012.05.012.

Orr H. Allen. 1991. Is single gene speciation possible? Evolution Vol. 45. No. 3. 764769. $10.2307 / 2409927$ $14,675-679$.

Phadnis Nitin and Orr H. Allen. 2009. A Single Gene Causes Both Male Sterility and Segregation Distortion in Drosophila Hybrids. Science Vol. 323. 376-379 
991

992

993

994

995

996

997

998

999

1000

1001

1002

1003

1004

1005

1006

1007

1008

1009

1010

1011

1012

1013

1014

1015

1016

1017

1018

1019

1020

1021

1022

1023

1024

1025

1026

1027

1028

1029

1030

1031

1032

1033

1034

1035

1036

1037

1038

1039

1040

1041

1042

1043

1044

1045

doi:10.1126/science.1163934

Palopoli M.F. and Wu C-I. 1994 Genetics of hybrid male sterility between Drosophila sibling species: A complex web of epistasis is revealed in interspecific studies. Genetics. 138, 329-341. DOI: 10.1534/genetics.106.067314.

Perez Daniel., Wu Chung-I. 1995 Further characterization of the Odyssm Llcus of hybrid sterility in Drosophila: One Gene is Not Enough. Genetics 140, 201-206.

Presgraves C. D., Balagopalan L., Abmayr M. S. and Orr H. A. 2003 Adaptive evolution drives divergence of a hybrid inviability gene between two species of Drosophila. Nature. 423, 715-719. DOI:10.1038/nature01679.

Presgraves C. Daven 2007 Speciation Genetics: Epistasis, Conflict and the Origin of Species Cellpress. Volume 17. Issue 4. Pag. R125-R127.

DOI:https://doi.org/10.1016/j.cub.2006.12.030

Rieseberg Loren H. and Blackman Benjamin K. 2010. Genes in evolution: the control of diversity and speciation. Speciation genes in plants. Annals of Botany 106: 439-455, 2010

doi:10.1093/aob/mcq126.

Rodriguez-Trelles F., Alarcón L. and Fontdevila, A. 2000 Molecular evolution and phylogeny of the buzzatii complex (Drosophila repleta group): a maximum-likelihood approach. Mol.biol.evol. 17, 1112-1122.

Ruiz A., Fontdevila A. and Wasserman M. 1982 The evolutionary history of Drosophila buzzatii III. The cytogenetic relationship between two sibling species of buzzatii cluster. Genetics. 101, 503-518.

Ruiz Alfredo and Wasserman Marvin. 1993. Evolutionary cytogenetics of the Drosophila

buzzatii species complex. Heredity 70 582-596. https://doi.org/10.1038/hdy.1993.85

Sawamura K., Davies W. A. and Wu C-I. 2000 Genetic analysis of speciation by means of introgression into Drosophila melanogaster. PNAS. 97, 2652- 2655. DOI: 10.1073/pnas.050558597.

Sawamura K., Roote J., Wu C-I. and Yamamoto M-T. 2004 Genetic Complexity Underlying Hybrid Male Sterility in Drosophila. Genetics. 166, 789-796.

Schaeffer Stephen W., Bhutkar Arjun., McAllister Bryant F., Matsuda Muneo., Matzkin Luciano M., O'Grady Patrick M., et al. 2008 Polytene Chromosomal Maps of 11 Drosophila Species: The Order of Genomic Scaffolds Inferred From Genetic and Physical Maps. Genetics 179: 1601-1655.

Templeton A. R. 1981 Mechanisms of speciation: a population genetic approach. Ann. Rev. Ecol. Syst. 12, 23-48.

Thurmond J, Goodman JL, Strelets VB, Attrill H, Gramates LS, Marygold SJ, Matthews BB, Millburn G, Antonazzo G, Trovisco V, Kaufman TC, Calvi BR and the FlyBase Consortium. FlyBase 2.0: FB2020_05, released Oct 14, 2020. The the Next generation. Ácidos nucleicos Res. 47 (D1) D759 - D765).

Ting C-T., Tsaur S-C., Wu M-L., and Wu C-I. 1998 A rapidly evolving homeobox at the site of a hybrid sterility gene. Science. 282, 1501-1504. DOI: 10.1126/science.282.5393.1501

Vos P., Hogers R., Bleeker M., Reijans M., Van de Lee Hornes M., Frijters A., et al. 1995 AFLP: a new technic for DNA fingerprinting. Nucleic Acid Res. 23, 4407-4414.

Wharton L. T. 1942 Analysis of the repleta group of Drosophila. Univ. Texas Publ. 4228, 23-52.

Wu C-I. \& Palopoli M.F. 1994 Genetics of post-mating reproductive isolation in animals. Annu. Rev. Genet. 28, 283-308.

Yamamichi Masato., and Sasaki Akira. 2013. Single-gene speciation with pleiotropy: effects of allele dominance, population size, and delayed inheritance. Evolution 67-7: 2011-2023. doi:10.1111/evo.12068

Song Xiang, Goicoechea Jose Luis, Ammiraju Jetty S. S., Luo Meizhong, He Ruifeng, Lin Jinke, Lee So-Jeong, Sisneros Nicholas, Watts Tom, Kudrna David A., Golser Wolfgang, Ashley Elizabeth, Collura Kristi, Braidotti Michele, Yu Yeisoo, Matzkin Luciano 
1046 M., McAllister Bryant F., Markow Therese Ann and Wing Rod A. 2011. The 19 Genomes 1047 of Drosophila: A BAC Library Resource for Genus-Wide and Genome-Scale 1048 Comparative Evolutionary Research. Genetics 187: 1023-1030 (April 2011). DOI: 1049 10.1534/genetics.111.126540 


\section{Figures}

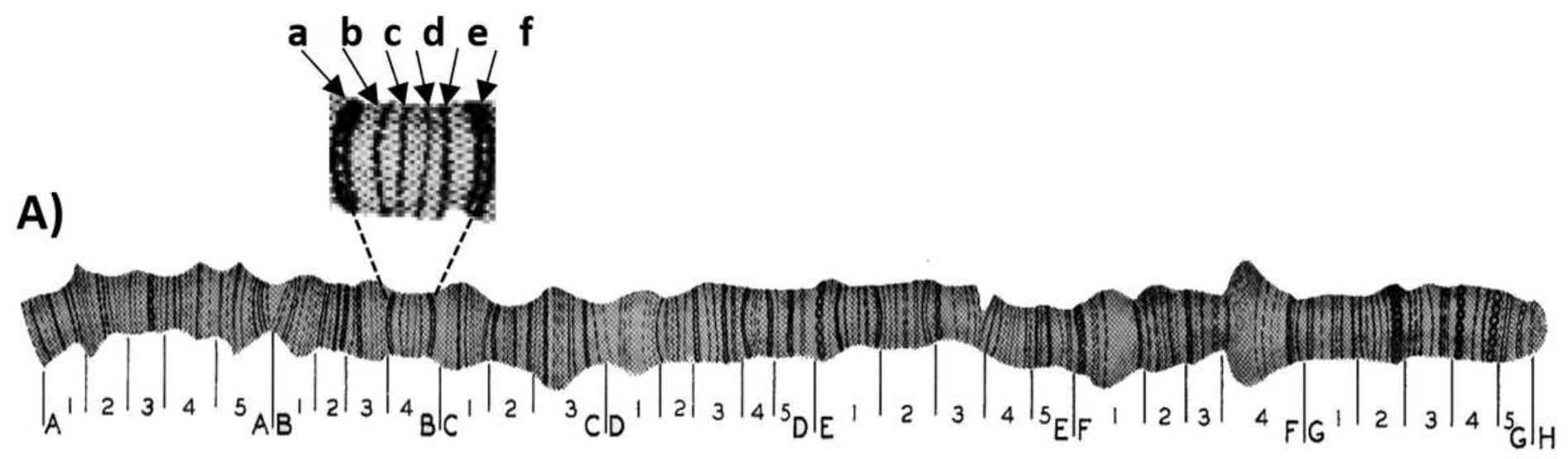

Figure 1

A) Cytological map of polytene chromosome 4 of Drosophila Repleta group species. The vertical lines divide each cytogenetic interval $(A$ to $H$ ) into subintervals identified by Arabic numerals 1 to 5 . The arrows show one example of the classification of bands identified by lowercase letters ( $a$ to $h$ ) to subinterval 4 into the cytological interval B. 


\begin{tabular}{|c|c|c|c|c|}
\hline No. & $\begin{array}{l}\text { Parents' } \\
\text { generation }\end{array}$ & $\begin{array}{l}\text { Type of } \\
\text { croos }\end{array}$ & Cross description & Offspring \\
\hline 1 & Parental & Mass & $\left(509 D k o \times 50 \sigma^{\prime} D b\right) \times 5$ & \\
\hline 2 & $\mathrm{BC}_{1}$ & Mass & $\left(50\right.$ 우 $\left.\mathrm{F} 1 \times 50 \sigma^{7} D b\right) \times 5$ [5] & F2 \\
\hline 3 & $\mathrm{BC}_{2}$ & Individual & $\left(19 \mathrm{~F} 2 \times 1 \sigma^{7} D b\right) \times 25$ [5] & F3 \\
\hline 4 & $\mathrm{BC}_{3}$ & Individual & $\left(1\right.$ 우 $\left.\mathrm{F} 3 \times 1 \sigma^{\prime} D b\right) \times 20$ [5] & F4 \\
\hline 5 & $\mathrm{BC}_{4}$ & Individual & $\left(19 \mathrm{~F} 4 \times 10^{7} \mathrm{Db}\right) \times 20$ [5] & \\
\hline$\stackrel{1}{14}$ & $\mathrm{BC}_{13}$ & Individual & $\left(1 \stackrel{5}{ } 13 \times 30^{\circ} D b\right) \times 20$ [5] & \\
\hline 15 & $\mathrm{BC}_{14}$ & Individual & $\left(19 \mathrm{~F} 14 \times 30^{\circ} D b\right) \times 20[5]$ & $\begin{array}{l}\text { F15 } \\
\mathbf{1}\end{array}$ \\
\hline 24 & $\mathrm{~B}^{\mathrm{B}} \mathrm{C}_{15}$ & Individual & $\left(19 \mathrm{~F} 23 \times 3 \sigma^{7} D b\right) \times 20[5]$ & $\mathrm{F} 24$ \\
\hline 25 & $\mathrm{BC}_{16}$ & Individual & $\left(1 \sigma^{\prime} \mathrm{F} 4 \times 39 D b\right) \times 20[5]$ & $\mathrm{F} 25$ \\
\hline \multicolumn{5}{|c|}{$\begin{array}{l}\text { BC: Backcross. } \\
\text { HC: Hybrid cross. } \\
\text { F: Filial offspring. } \\
\text { Db: Parental strain of Drosophila buzzatii. } \\
\text { Dko: Parental strain of D. koepferae. } \\
\text { [ ] Number of replicates of each series. }\end{array}$} \\
\hline
\end{tabular}

Figure 2

Crossbreeding protocol to obtain single-segmental lines: 1) obtaining hybrid offspring; 2) recombinant offspring; 3) multi-segmental offspring in all chromosomes; 4) multiple segmental offspring just in the four chromosome; 5 to 14) selection of single segmental lines exclusively in the four chromosome; 15 to 24) Just single segmental lines with segments of interest; 25) lines in stock. 


\section{Crosses to obtain segmental homozygous offspring}

Generation

Crossing

Offspring

Offspring

Crossing

Generation

1)

$\mathrm{BC}_{1}$ : line B1 $10^{\circ}$ line $\mathrm{B} 1 \times 1$ 우 Db F2

(B1/bu)

$\mathrm{A}\left(\sigma^{\circ} \mathrm{bu} / \mathrm{bu} X\right.$ obu/bu) $\mathrm{F} 2 \mathrm{~A}(\mathrm{bu} / \mathrm{bu})$

$F 2_{R}$

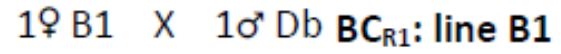

(bu/bu)

$\mathrm{B}\left(\mathrm{o}^{\mathrm{B}} \mathrm{B} 1 / \mathrm{bu}, \mathrm{X}+\mathrm{bu} / \mathrm{bu}\right) \longrightarrow \mathrm{F} 2 \mathrm{~B}(\mathrm{~B} 1 / \mathrm{bu}, \mathrm{bu} / \mathrm{bu})$

F2A $(b u / b u)$

4

$\mathrm{F}_{2} \mathrm{~B}_{\mathrm{R}}(\mathrm{B} 1 / \mathrm{bu}, \mathrm{bu} / \mathrm{bu})$

$4($ B1/bu, $\mathrm{X}$ ơbu/bu) B

(B1/bu)

(bu/bu)

Isolation of

2) Isolation of male $\mathrm{C} 1$ parents

3) Genotypes of parents $\mathrm{BC}_{1}$

$\mathrm{HC}_{1}$ : heterozygous

4) segmental parents (B1/bu) from $\mathrm{BC}_{1}$ and $\mathrm{BC}_{\mathrm{R} 1}$

$\sigma^{\prime B} 1 / b u \quad x \%(B 1 / b u)$

Offspring F3 (segmental homozygous 1 BB1) FIRS

female $\mathrm{C} 1$

parents

Genotypes of

Parents $\mathrm{BCR}_{1}$

(B1/B1, B1/bu, bu/bu)

5) $\begin{gathered}\mathrm{BC}_{2} \\ \text { To fertility } \\ \text { evaluation one }\end{gathered}$

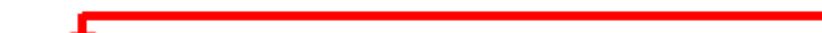

\begin{tabular}{|c|c|c|c|}
\hline F3 & $\mathrm{X}$ & O Db & F4 \\
\hline (bu/bu & $x$ & $\mathrm{bu} / \mathrm{bu})$ & $\mathrm{F} 4 \mathrm{~A}(\mathrm{bu} / \mathrm{bu})$ \\
\hline (B1/bu & $\mathrm{x}$ & $\mathrm{bu} / \mathrm{bu})$ & $\mathrm{F} 4 \mathrm{~B}(\mathrm{~B} 1 / \mathrm{bu}, \mathrm{bu} / \mathrm{bu})$ \\
\hline (B1/B1) & $\mathrm{x}$ & bu/bu) & $\mathrm{F} 4 \mathrm{C}(\mathrm{B} 1 / \mathrm{bu})$ \\
\hline
\end{tabular}

6)

$\mathrm{HC}_{1}$ : heterozygous

$\mathrm{o}^{\mathrm{B}} \mathrm{B} 1 / \mathrm{bu} \times$ ㅇ $\mathrm{B} 1 / \mathrm{bu}$ segmental (B1/bu)

from $\mathrm{F} 4_{R} \mathrm{C}$ )

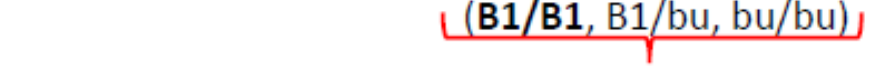

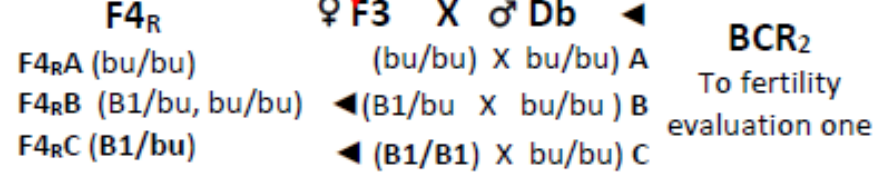

I

Offspring F5 (segmental homozygous 2 BB1)

(B1/B1, B1/bu, bu/bu)
$\mathrm{BC}_{3}$
To fertility evaluation two
F6
$\mathrm{A}(\mathrm{bu} / \mathrm{bu} \times \mathrm{bu} / \mathrm{bu}) \quad \mathrm{F} 5 \mathrm{~A}(\mathrm{bu} / \mathrm{bu})$
B (B1/bu $X$ bu/bu)
F5B (B1/bu, bu/bu)
$\mathrm{C}(\mathrm{B} 1 / \mathrm{B} 1) \times \mathrm{bu} / \mathrm{bu}) \longrightarrow \mathrm{F} 5 \mathrm{C}(\mathrm{B} 1 / \mathrm{bu})$

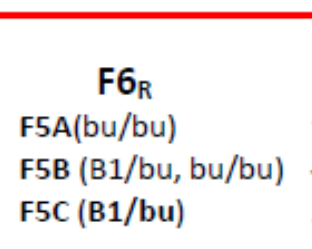
\% F5 $\times$ o $\mathrm{Db}$
4 (bu/bu) X bu/bu) A
$\mathrm{BCR}_{3}$
(B1/bu X bu/bu ) B To fertility
4 (B1/B1) $x$ bu/bu) C evaluation two

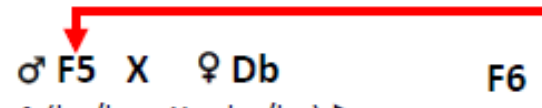

BC: Backcross

$\mathrm{BC}_{\mathrm{R}}$ : Reciprocal backcross

HC: Hybrid cross

F: Filial offspring

B1: segmental heterozygous line

BB1: Segmental homozygous offspring

Dbu: Parental strain of Drosophila buzzatii

bu/bu: Genotype of parental Db

B1/bu: Genotype of heterozigous segmental

B1/B1: Genotype of Homozygous segmental

Figure 3

General scheme to obtaining segmental offspring in homozygosity.

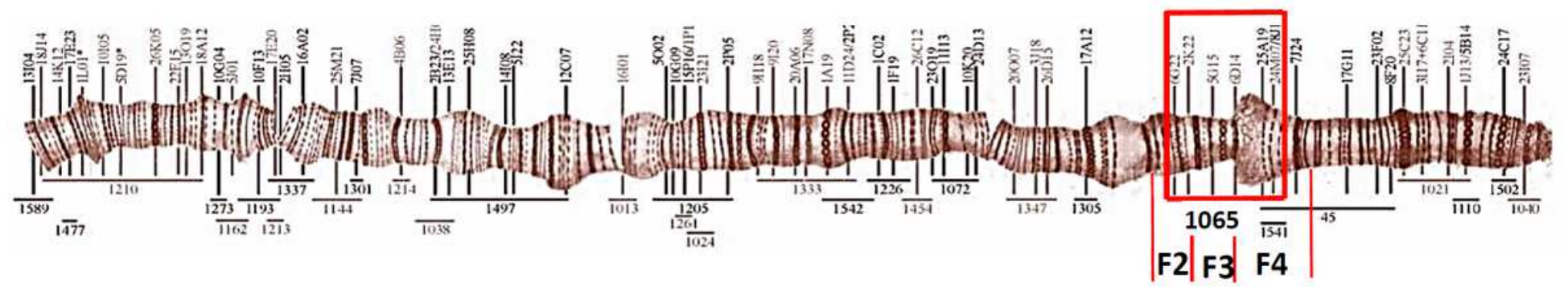


Integrated BAC-based physical map of the Drosophila buzzatii genome. Vertical lines indicate the relative position of the each of the $65 \mathrm{BAC}$ clones label. The 31 contigs to which the hybridized clones belong are represented by short horizontal lies below the chromosomes along with the respective contig number. Into the red rectangle, the position of contig 1065 is indicated where the chromosomal fragments of interest F2, F3, and F4 are located. (Modified image from Gonzalez et al, 2005).

50

\section{Parents by genotype and sex}

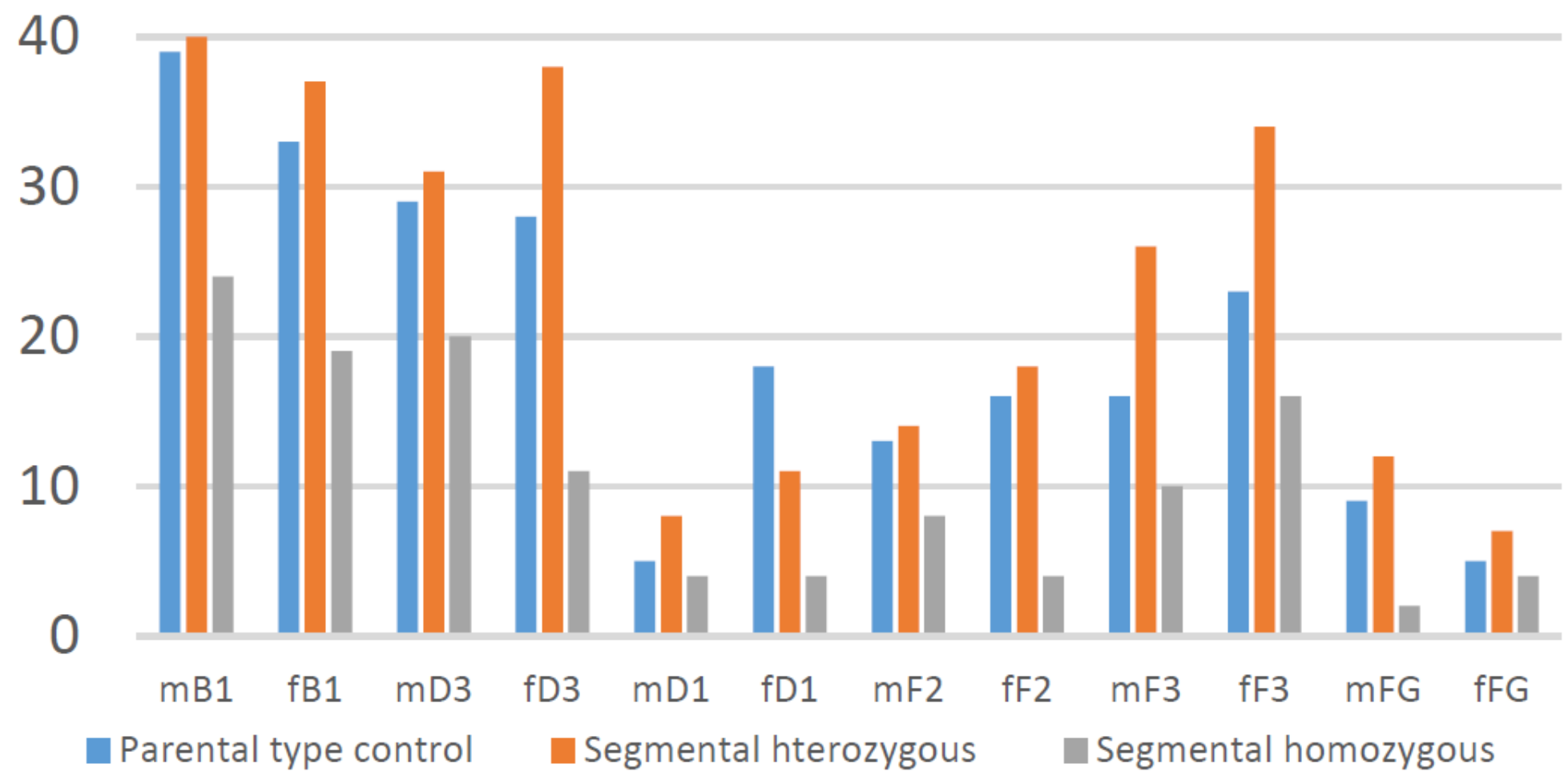

\section{Figure 5}

Offsprings grouped according to their introgressive segment (B1, D3, D1, F2, F3 and FG), and their sex ( $m$ $=$ male, $f=$ female) . 


\section{Male and females parents by genotype}

\section{0}
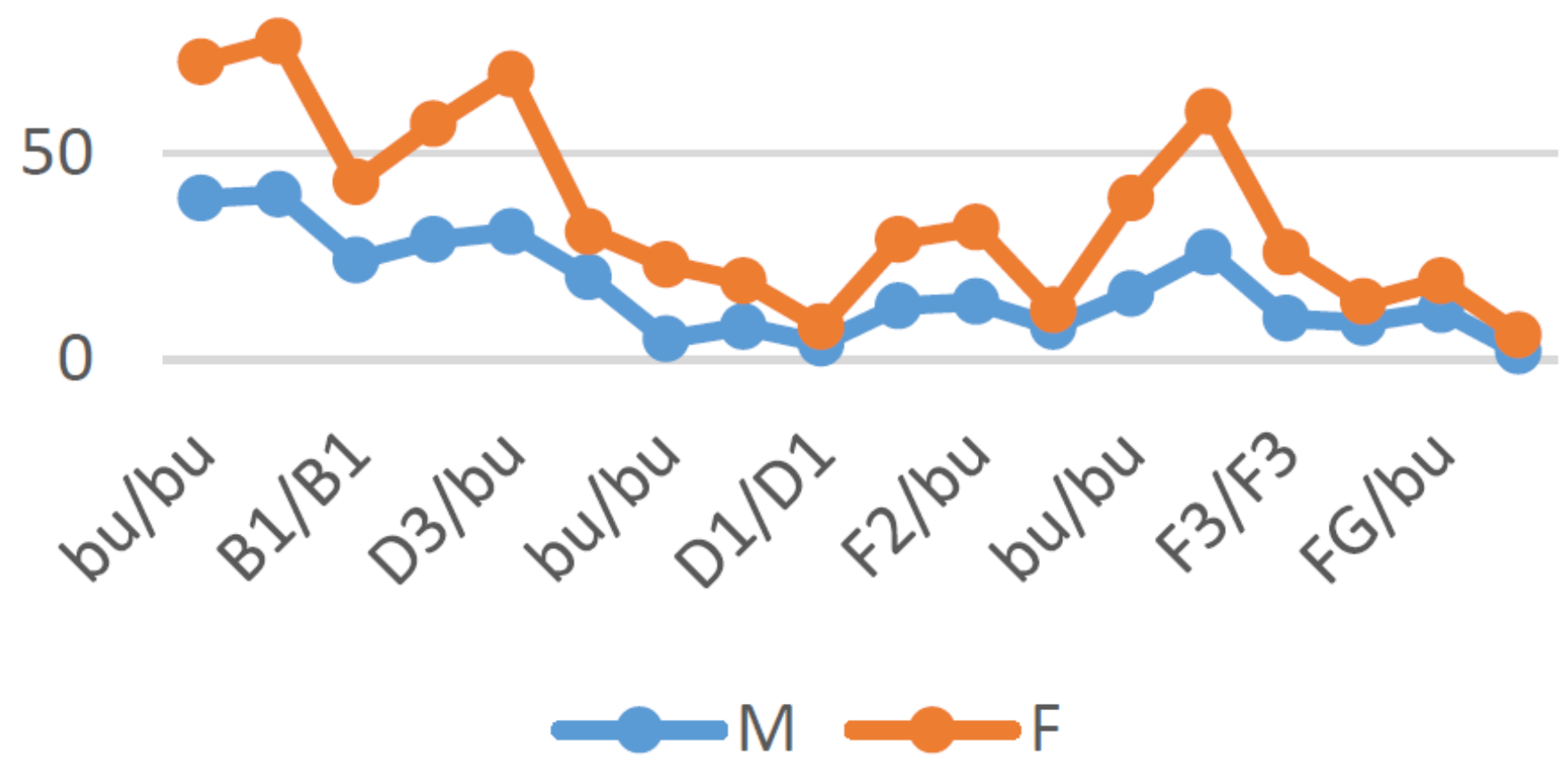

Figure 6

Comparison of the number of male and female parents of each type of offspring (B1, D3, D1, F2, F3, and FG) according to their three respective genotypes, parental, segmental heterozygous, and segmental homozygous. 


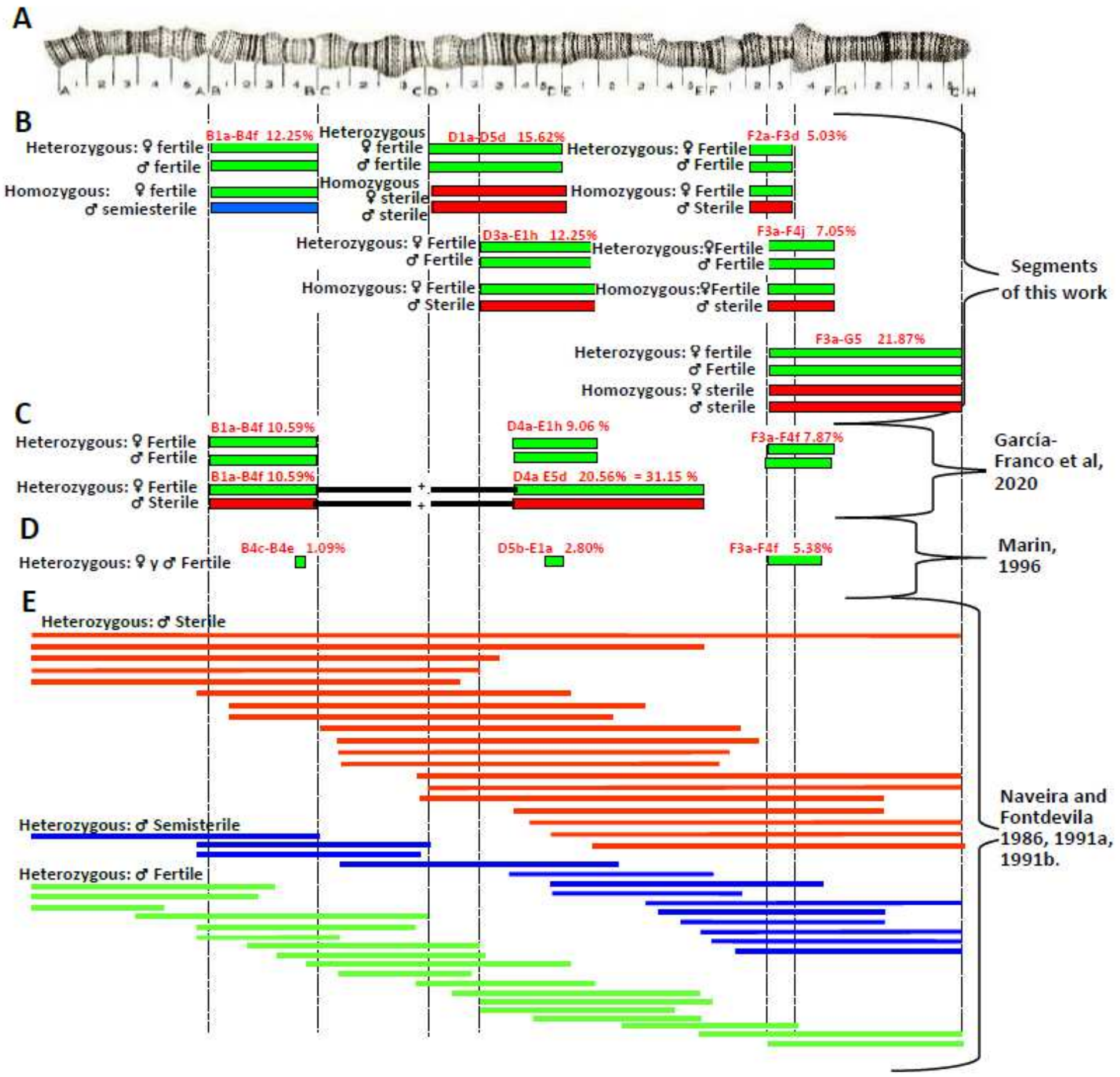

Figure 7

(A) Total length of chromosome 4 of the Repleta group. (B) Representation of the 6 segments evaluated in heterozygosity and the 6 segments evaluated in homozygosis in this work. (C) representation of segments evaluated in heterozygosity in García-Franco et al, 2020. (D) representation of segments evaluated in heterozygosity Marin, 1996. (E) representation of segments evaluated in heterozygosis in Naveira and Fontdevila 1986, 1991a, 1991b. Each bar of a certain color represents each chromosomal segment, and each color indicates a level of sterility or fertility that each segment produces: Green fertile 
Blue: semi-sterile Red: sterile. The name and size of each segment can be seen at the top of its respective segment.

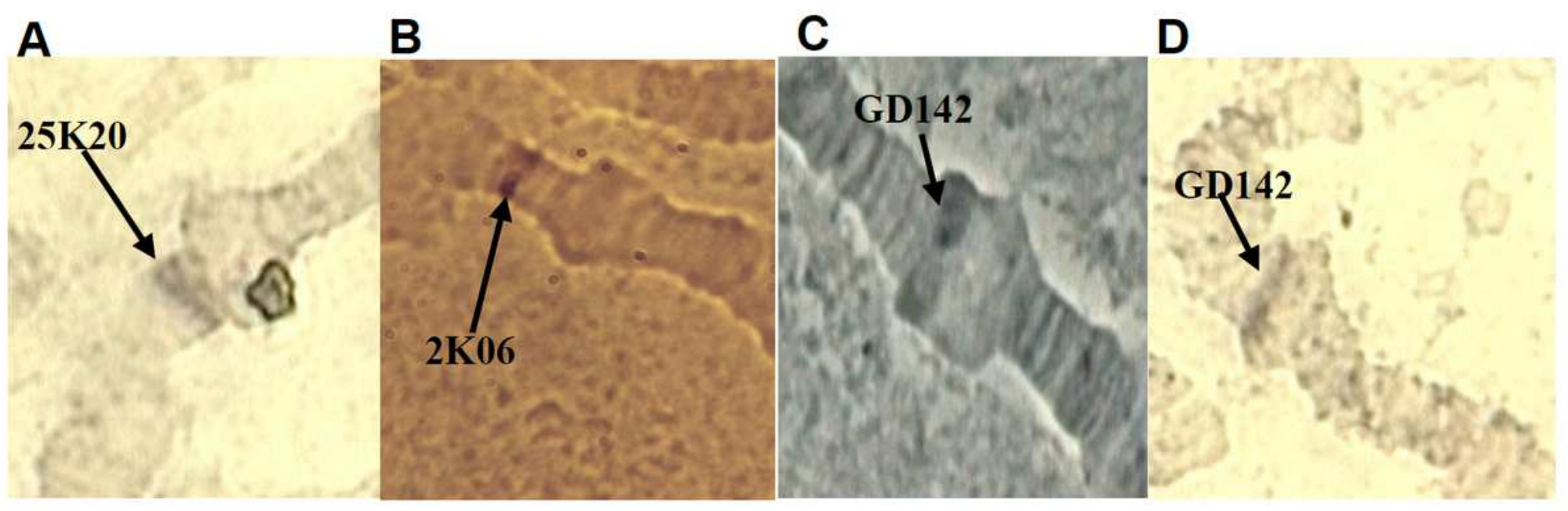

Figure 8

Positive signals from in situ hybridizations. A) and B) positive signals from probes $25 \mathrm{~K} 20$ and $2 \mathrm{~K} 06$ in D. buzzatii. C) GD142 probe in D. buzzatii. D) GD142 probe in D. mojavensis.

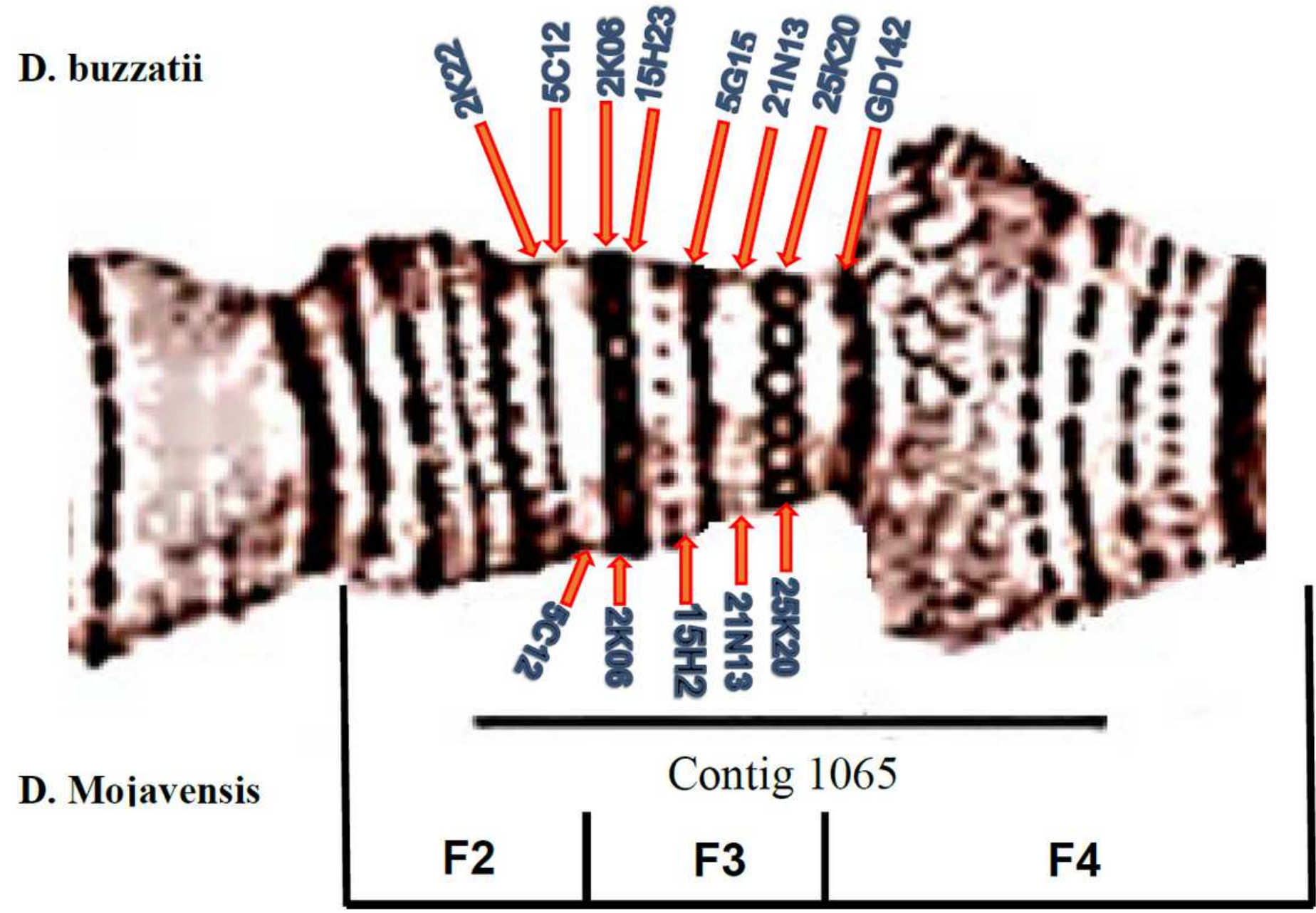




\section{Figure 9}

In situ hybridizations of 8 BACs clones in D. buzzatii and D. mojavensis. The arrows in the upper part corresponding to the hybridizations in polygenic chromosomes of D. buzzatii, and the arrows in the lower part those of D. mojavensis. Each arrow indicates the position of the hybridization signal corresponding to the respective probe according to the indicated key. The black line under the chromosome image represents the full length of contig 1065. The 3 regions F2, F3 and F2 of chromosome 4 are indicated.

A) Drosophila buzzatii Genome Project (DBGP)

D. buzzatii-chr_4. Scaffold2, contig 01065, length $=4233$ nt (308944 a 313176)

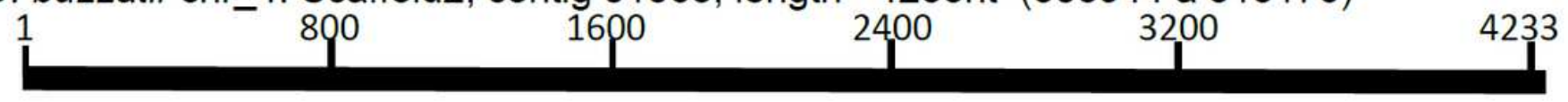

B) GenBAnK. BLAST. Job title: Contig 01065 length= 4233

Optimize for Highly similar sequences (megablast)

D. melanogaster-chr3R (28074717-28065867)

C) FlyBase. BLAST. Contig01065 length $=4233 \mathrm{bp}$

BLAST 3 Dmoi scaffold 6540 (19163410-19 167114)

Figure 10

Schematic representation of the homologous sequences identified by the Blast procedure, between Contig 1065 and the nucleotide sequences of the species of the genus Drosophila. A) Contig 1065 of 4233 nucleotides in total length. B) Eleven homologous segments between the D. melanogaster sequences and the 1065 contig. C) Twenty homologous segments between the D. mojavensis sequences and the 1065 contig. Each rectangle indicates a homologous segment between the respective Drosophila species with the 1065 contig of D. buzzatii.

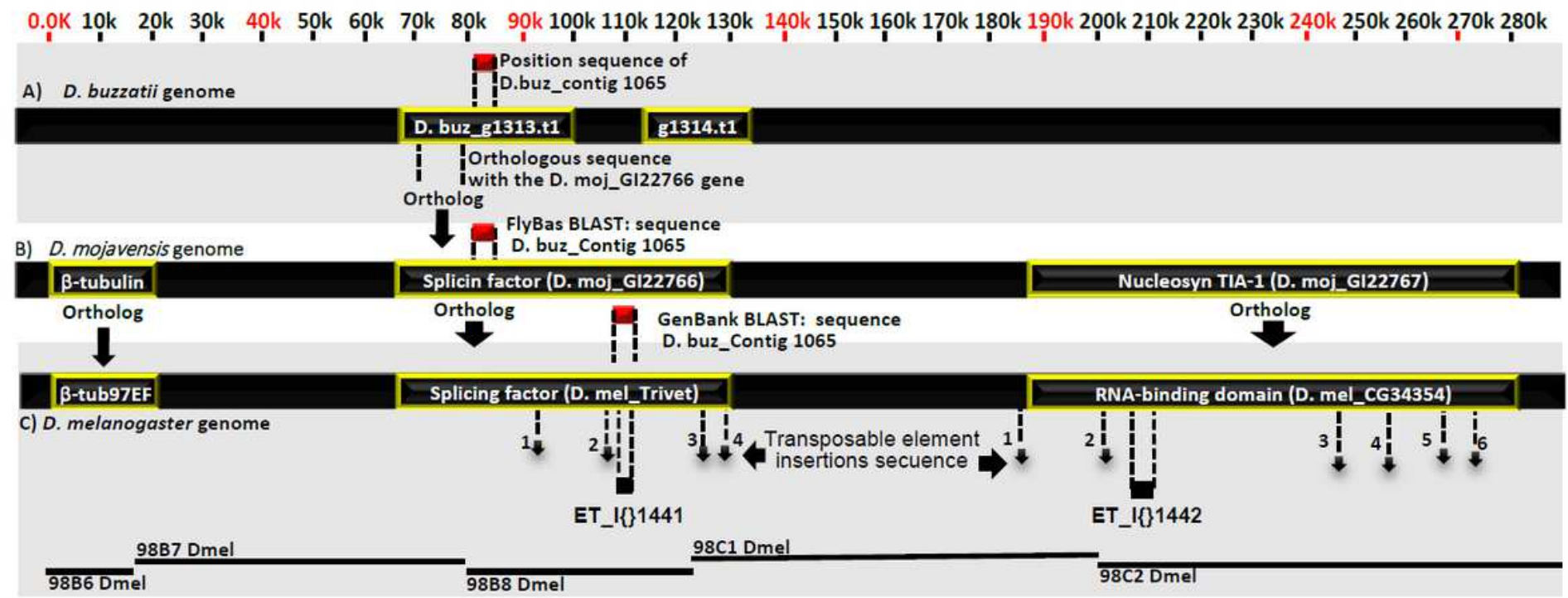




\section{Figure 11}

Genetic regions of the genomic maps. A) D. buzzatii; B) D. Mojavensis and C) D. melanogaster, which are associated with the nucleotide sequence of contig 1065 of D. buzzatii. The numerical scale in kilobases at the top indicates only the relative distance of each genetic element on its respective genomic map. The long black lines indicate the genomic region represented, respective of each species. Black rectangles with yellow margins represent the position of each of the indicated genes. The small rectangles in red color indicate the positions of the homologous sequences between the 1065 contig with the genome of $D$. buzzatii, D. mojavensis and D. melanogaster respectively. The smaller black rectangles at the bottom locate the natural transposable elements of each gene. The numbers 1 to 6 indicate the presence of the mobile element insertion sequences and the lower black lines represent the regions of the cytogenetic bands $98 \mathrm{~B} 6$ to $98 \mathrm{C} 2$ of the cytogenetic map of D. melanogaster. 